\title{
Originalarbeiten
}

\section{Humanarzneimittel im aquatischen Ökosystem}

\section{Bewertungsansatz zur Abschätzung des ökotoxikologischen Risikos von Arzneimittelrückständen}

\author{
Bernd Hanisch $^{1 *}$, Bettina Abbas ${ }^{1}$, Werner Kratz ${ }^{1}$ und Gerrit Schüürmann ${ }^{2}$ \\ ${ }^{1}$ Landesumweltamt Brandenburg, Müllroser Chaussee 50, D-15326 Frankfurt (Oder) \\ 2 UFZ-Umweltforschungszentrum Leipzig-Halle GmbH, Permoser Straße 15, D-04318 Leipzig
}

* Korrespondenzautor (bernd.hanisch@lua.brandenburg.de)

\section{DOI: http://dx.doi.org/10.1065/uwsf2004.02.076}

\section{Zusammenfassung}

Zielstellung. Im Landesumweltamt Brandenburg wurde auf der Grundlage einer für das Jahr 1999 durchgeführten landesspezifischen Verbrauchsmengenerhebung ein Bewertungsansatz für das Gefährdungspotenzial von Arzneimittelrückständen im aquatischen Ökosystem mit der Zielstellung entwickelt, eine mögliche Umweltrelevanz wichtiger Wirkstoffe festzustellen oder auszuschließen sowie prioritäre Analyten für künftige Monitoringprogramme zu definieren.

Methode. Dieses an 60 Arzneimittelwirkstoffen erprobte Bewertungskonzept basiert auf einer Expositionsabschätzung unter Berücksichtigung des Haupteintragspfades Mensch-AbwasserKläranlage-Oberflächengewässer, auf einer stoffspezifischen Wirkungsanalyse sowie auf einer Analyse bekannter Umweltverhaltensmuster wie Kompartimentverteilungstendenzen, Bioakkumulationsvermögen und Persistenz.

Ergebnisse. Während in Brandenburger Oberflächengewässern für acht der betrachteten Wirkstoffe von Wirkstoffkonzentrationen oberhalb von $1 \mu \mathrm{g} / \mathrm{l}$ auszugehen ist, liegt für mindestens 13 der untersuchten Wirkstoffe die PNEC im aquatischen Ökosystem unter $1 \mu \mathrm{g} / \mathrm{l}$.

Für die Antibiotika Ciprofloxacin- $\mathrm{HCl}$ und Clarithromycin, die Desinfektionsmittelwirkstoffe Benzalkoniumchlorid, Cocospropylendiaminguaniacetat, Glucoprotamin, Laurylpropylendiamin und Polyvidon-Iod, das Sexualhormon Ethinylestradiol, das Antidiabetikum Metformin- $\mathrm{HCl}$, das Antiepileptikum Carbamazepin sowie für den Lipidsenkermetaboliten Clofibrinsäure lässt sich anhand des Vergleiches von Expositionskonzentrationen und ökotoxischen Wirkungen ein Umweltgefährdungspotential für Brandenburger Oberflächengewässer ableiten, da die entsprechenden PEC:PNEC-Verhältnisse um 1 oder darüber liegen.

Für weitere 19 Wirkstoffe muss aufgrund ihrer Verhaltensmuster in der Umwelt ebenfalls von einer Umweltrelevanz ausgegangen werden, ohne dass diese derzeit wegen des Fehlens von ökotoxikologischen Wirkungsdaten wirkungsseitig belegt werden kann.

Schlussfolgerung. Diese erste Risikoabschätzung zeigt trotz einer unvollständigen Basis valider Daten, dass Schadwirkungen in der aquatischen Umwelt durch einige Arzneimittelwirkstoffe nicht auszuschließen sind. Es ist uns jedoch bewusst, das diese Bewertung zunächst orientierenden Charakter hat und im Zuge zu erwartender und zu fordernder neuer wissenschaftlicher Erkenntnisse fortund möglicherweise auch umgeschrieben werden muss.

Schlagwörter: Aquatisches Ökosystem; Expositionsanalyse; Gefährdungsabschätzung; Humanarzneimittel; Land Brandenburg; Oberflächengewässer; Ökotoxikologie; ökotoxikologische Bewertung; Wirkungsanalyse

\section{Abstract \\ Human Drugs in Aquatic Ecosystems: Approach for the Environmental Risk Assessment of Drug Residues}

Goal. In the Brandenburg State Office for the Environment an approach to assess the potential risk of drug residues in aquatic ecosystems has been developed based on an investigation of the consumption amounts in 1999 to identify or to exclude a potential environmental risk of important human drugs and further to define priority substances for monitoring programs in Brandenburg in the future.

Method. This assessment approach tested with 60 drug agents is based on an exposure estimation considering the main path for the entry human - waste water - sewage plant - surface water, on a substance specific analysis of effects and on an analysis of the environmental fate as compartment distribution, bioaccumulation and persistence. Results. In Brandenburg surface waters the drug agent concentration for eight of the considered substances is supposed to be greater than $1 \mu \mathrm{g} /$ and for at least 13 of the considered substances the PNEC in aquatic ecosystems is assumed to be lower than $1 \mu \mathrm{g} /$ l.

A potential risk for the environment can be seen for the antibiotics Ciprofloxacin- $\mathrm{HCl}$ and Clarithromycin, the disinfectants Benzalkonium Chloride, Cocospropylendiaminguaniacetat, Glucoprotamine, Laurylpropylenediamine and Polyvidone-iodine, the sexual hormon Ethinylestradiol, the antidiabetic Metformin- $\mathrm{HCl}$, the antiepileptic Carbamazepine and the lipid regulator Clofibrinic acid on the basis of effects because their PEC:PNEC-relation is about 1 or higher. For further 19 drug agents an environmental risk is to be assumed because of their environmental behaviour without support by ecotoxicological data at present.

Conclusion. Although there is only a small base of valid ecotoxicological data, this risk assessment shows that adverse effects in the aquatic environment by some drug agents cannot be excluded. However, it should be noted that this first assessment gives only a crude orientation. Appropriate test data are necessary to refine the assessment in future.

Keywords: Aquatic ecosystem; ecotoxicological assessment, effect analysis; ecotoxicology; exposure analysis; human pharmaceuticals; Land Brandenburg; risk assessment; surface water

\section{Einleitung und Zielstellung}

Entsprechend der Definition des Arzneimittelgesetzes sind Arzneimittel u.a. dazu bestimmt, Krankheiten und Leiden zu heilen, zu lindern, zu verhüten oder zu erkennen. Diese unbestritten positive Zweckbestimmung ließ bis in die jüngste Vergangenheit, von möglichen unerwünschten Nebenwirkungen im Zielorganismus und Fragen der Kostenbudgetierung 
einmal abgesehen, wenig Raum, den nicht unbeträchtlichen Arzneimittelkonsum in Deutschland beispielsweise auch unter dem Gesichtspunkt der Umweltverträglichkeit kritisch zu betrachten. Eine veränderte Situation ergab sich diesbezüglich, als in den neunziger Jahren vermehrt Arzneimittelwirkstoffe bzw. deren Metaboliten in Oberflächen-, Grundund Trinkwässern nachgewiesen wurden [1-4].

Da Arzneimittelwirkstoffe als biologisch hochaktive Stoffe teilweise in ähnlichen Konzentrationen wie Pflanzenschutzmittel in der aquatischen Umwelt anzutreffen sind, ergibt sich die Frage nach eventuellen Schadwirkungen auf Nichtzielorganismen im aquatischen Ökosystem nahezu zwangsläufig.

Eine Bewertung derartiger Schadwirkungen erfordert einerseits Angaben zu Konzentrationen im jeweils betrachteten Umweltkompartiment sowie andererseits die Kenntnis von Wirkschwellen bzw. Effektkonzentrationen, oberhalb derer mit schädigenden Wirkungen gerechnet werden muss. Um zu einer vorläufigen Risikobewertung zu gelangen, kann dabei auch auf Abschätzmethoden zurückgegriffen werden.

Für eine erste grobe Gefährdungsabschätzung von Arzneimittelrückständen im aquatischen Ökosystem, nachfolgend exemplarisch für den Ausbreitungspfad Mensch-kommunales Abwasser-Kläranlage-Oberflächengewässer durchgeführt, ergaben sich somit folgende Notwendigkeiten:

- aufbauend auf der vom Landesumweltamt Brandenburg [4,5] für das Jahr 1999 durchgeführten landesspezifischen Erhebung mengenmäßig relevanter Arzneimittelwirkstoffe und ersten in Brandenburger Oberflächengewässern analytisch bestimmten Wirkstoffkonzentrationen eine Predicted Environmental Concentration für Oberflächengewässer des Landes Brandenburg, (PEC-BRB) zu ermitteln. Humanmetabolismus und Kläranlagenelimination sollten dabei für jeden Wirkstoff berücksichtigt werden

- ökotoxikologische Wirkungsdaten für wichtige im Land Brandenburg applizierte Humanarzneimittel zu sammeln und aus diesen mit Hilfe von Sicherheitsfaktoren einen stoffspezifischen Schwellenwert für schädliche Wirkungen im aquatischen Ökosystem zu definieren (Ableitung einer Predicted No Effect Concentration, PNEC)

- durch Vergleich von PEC und PNEC sowie unter Berücksichtigung von Akkumulationsverhalten, Persistenz, biologischer Abbaubarkeit und chronischer Human- und Säugertoxizität eine mögliche Umweltrelevanz für das aquatische Ökosystem festzustellen oder auszuschließen, sowie

- unter landesspezifischen Gesichtspunkten prioritäre Analyten für Oberflächengewässer zu identifizieren.

\section{Bewertungsmethode}

Als Hauptelemente der durchgeführten Abschätzung des ökotoxikologischen Risikopotentials sind neben einer vorab erfolgten Auswahl relevanter Wirkstoffe (siehe 1.1) eine ori- entierende Abschätzung der Exposition im Oberflächenwasser (siche 1.2) sowie eine Prognose möglicher Wirkungen auf das aquatische System (siehe 1.3) zu nennen. Die Bewertung der ökotoxikologischen Relevanz erfolgt dann, sofern ökotoxikologische Wirkungsdaten vorhanden sind, durch Vergleich von Exposition und Wirkung, genauer gesagt durch die Beantwortung der Frage, ob die für das Oberflächenwasser gemessenen oder berechneten Wirkstoffkonzentrationen kleiner, etwa gleich groß oder größer sind als die niedrigsten bekannten Effektkonzentrationen auf aquatische Organismen. Bei fehlenden Wirkungsdaten erfolgt eine Prognose anhand sonstiger Daten zum Umweltverhalten (siehe 1.4). Schließlich ergibt sich aus der Risikobewertung eine Empfehlung, ob und in welcher Form eine Analytik künftig erfolgen soll (siehe 1.5). Im einzelnen wird die Vorgehensweise durch Abb. 1 verdeutlicht.

\subsection{Wirkstoffauswahl}

Die Auswahl der zu bewertenden Wirkstoffe erfolgte zunächst nach dem Kriterium relevanter Verbrauchsmengen im Land Brandenburg. Dazu wurden aus den im Landesumweltamt Brandenburg [4,5] für das Jahr 1999 erhobenen landesspezifischen Verbrauchsmengen alle Wirkstoffe mit einer jährlichen Verbrauchsmenge $>250 \mathrm{~kg}$ (entspricht Input A in Abb. 1 bzw. lfd. Nr. 1-39 in Tab. 1) sowie Hauptvertreter von Stoffen mit besonderen Wirkmechanismen wie Antibiotika, Zytostatika, Hormone, Narkotika (entspricht Input B in Abb. 1 bzw. Ifd. Nr. 40-52 in Tabelle 1) selektiert. Alle ausgewählten Wirkstoffe sind in der nachfolgenden Tabelle 1 aufgelistet.

Eine dritte Auswahlgruppe (entspricht Input C in Abb. 1 bzw. Ifd. Nr. 53-60 in Tabelle 2) besteht aus Wirkstoffen, die nicht Bestandteil von Input A oder B sind, jedoch bei zwischenzeitlich erfolgten ersten analytischen Messungen in Brandenburger Oberflächengewässern (Messprogramm des BLAC [8], Messungen des LUA Brandenburg in der Schwarzen Elster, Messungen im Teltowkanal in Kleinmachnow [9]) oberhalb ihrer Bestimmungsgrenze nachgewiesen werden konnten.

\subsection{Expositionsanalyse}

Ziel der Expositionsanalyse (hier für die Wirkstoffe der InputGruppen A und B) war die Ermittlung einer für das Land Brandenburg spezifischen Predicted Environmental Concentration (PEC-BRB) für Oberflächengewässer unter Berücksichtigung der Kriterien jährliche Verbrauchsmenge des Wirkstoffes, Humanmetabolismus und Kläranlagenelimination. In Analogie zum Vorschlag im Diskussionspapier zur Abschätzung von Umweltrisiken durch Humanarzneimittel der EMEA [10] wurde in einem ersten Schritt zunächst eine PEC ${ }_{\text {wc }}$-BRB als worst case nach Gleichung (1) berechnet:

Jahresverbrauch in $\mathrm{kg} *[100-$ Rückhalterate in $\%] * 10^{4}$

$\mathrm{PEC}_{\mathrm{wc}}-\mathrm{BRB}[\mu \mathrm{g} / \mathrm{l}]=$

[365 $\mathrm{d}$ * Einwohnerzahl BRB * Abwasseranteil pro Kopf $\left(\mathrm{m}^{3 / \mathrm{d}}\right)$ * Verdünnung]
(2,6 Mio.)
$\left(0,15 \mathrm{~m}^{3} / \mathrm{d}\right)$
(10-fach) 


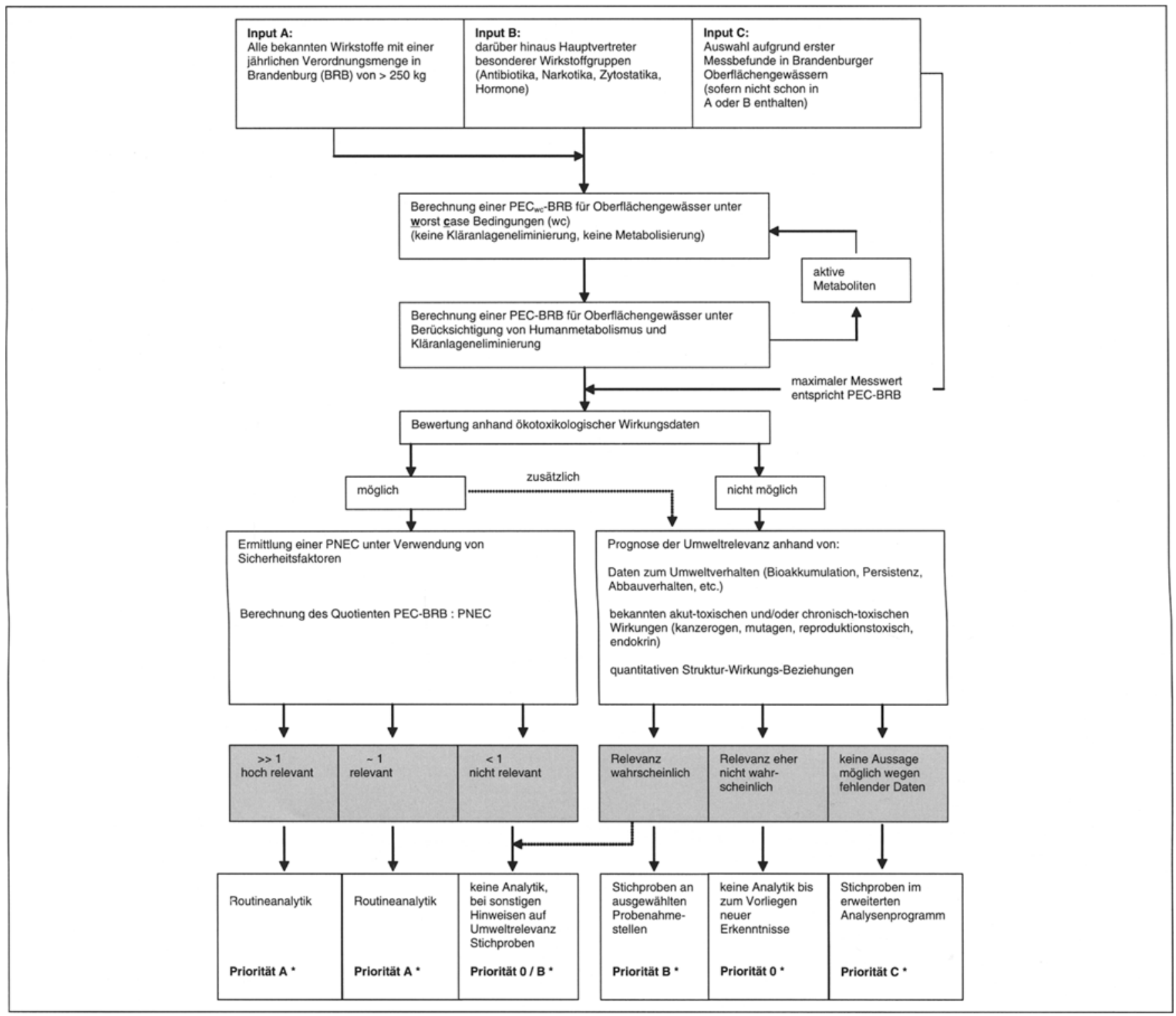

Abb 1: Verfahrensschema zur ökotoxikologischen Bewertung von Arzneimitteln ( ${ }^{\star}$ : zur Definition der Prioritäten siehe 1.5)

Bei dieser Abschätzung wird zunächst davon ausgegangen, dass die eingenommene Wirkstoffmenge unverändert wieder ausgeschieden wird, über das Abwasser ins Klärwerk gelangt und dort keine Eliminierung stattfindet (Rückhalterate $=0 \%$ ). Ferner wird von einer durchschnittlichen 10 fachen Verdünnung des Abwassers im Oberflächengewässer ausgegangen. Es werden dabei außerdem folgende vereinfachende, in der Praxis aber teilweise oft nicht zutreffende Annahmen getroffen:

- zeitlich gleichmäßige Verteilung der Einnahme und somit auch Ausscheidung über das gesamte Jahr

- örtliche Gleichverteilung im Oberflächengewässer

- kein späterer (z.B. photolytischer) Abbau im Oberflächenwasser

- der Eintragspfad über die Kläranlage stelit den alleinigen Eintragsplad dar

Für die nach der jährlichen Verbrauchsmenge ausgewählten Wirkstoffe (Input A) ergibt sich für die unterste Mengengrenze $(250 \mathrm{~kg} / \mathrm{a})$ nach der Berechnungsformel eine $\mathrm{PEC}_{\mathrm{wc}}{ }^{-}$ $\mathrm{BRB}$ von $0,2 \mu \mathrm{g} / \mathrm{l}$.
Aus der so grob errechneten $\mathrm{PEC}_{\mathrm{wc}}-\mathrm{BRB}$ wurde, sofern Daten zum Humanmetabolismus und zur Kläranlageneliminierung verfügbar waren, unter Berücksichtigung dieser Daten (Tabelle 3) eine genauere PEC-BRB für Brandenburger Oberflächengewässer nach Gleichung (2) berechnet:

$\mathrm{PEC}-\mathrm{BRB}=\mathrm{PEC}_{\mathrm{wc}}-\mathrm{BRB}$

* Anteil des unverändert ausgeschiedenen Wirkstoffes [\%] 100

* $100-$ Kläranlageneliminierung [\%]

$$
100
$$

Diese PEC-BRB fand für die Wirkstoffe nach Input A und B (außer für Metoprololtartrat, Diclofenac-Na und Indometacin) für die nachfolgende Bewertung eines möglichen Umweltrisikos als (geschätzte) Wirkstoffkonzentration in Oberflächengewässern des Landes Brandenburg Anwendung. 
Tabelle 1: Input A und B: Wirkstoffauswahl nach der jährlichen Verbrauchsmenge im Land Brandenburg (1999), Kriterium: $>250 \mathrm{~kg} / \mathrm{Jahr}$ sowie Hauptvertreter von Stoffen mit besonderen Wirkmechanismen

\begin{tabular}{|c|c|c|c|c|}
\hline Lfd. Nr. & Wirkstoff & Indikation & $\begin{array}{c}\text { Indikationsnummer } \\
\text { nach } \\
\text { Rote Liste [7] }\end{array}$ & $\begin{array}{c}\text { Jährliche } \\
\text { Verbrauchsmenge } \\
\text { in BRB in kg [5] }\end{array}$ \\
\hline 1 & Acetylsalicylsäure & Analgetikum & 5 & 24.269 \\
\hline 2 & Acetylcystein & Antitussivum & 24 & 10.561 \\
\hline 3 & Phenoxypropanol-Isomere ${ }^{a}$ & Desinfizientium / Antiseptikum & 33 & 7.892 \\
\hline 4 & Metformin- $\mathrm{HCl}$ & Antidiabetikum & 12 & 7.620 \\
\hline 5 & Paracetamol & Analgetikum & 5 & 6.753 \\
\hline 6 & Glucoprotamin $^{\text {a }}$ & Desinfizientium / Antiseptikum & 33 & 5.023 \\
\hline 7 & Iodixanol & Diagnostikum (Röntgenkontrastmittel) & 35 & 4.940 \\
\hline 8 & Metamizol-Na & Analgetikum & 5 & 4.649 \\
\hline 9 & Cocospropylendiaminguaniacetat $^{\mathrm{a}}$ & Desinfizientium / Antiseptikum & 33 & 3.157 \\
\hline 10 & Theophyllin & Antiasthmatikum & 28 & 2.790 \\
\hline 11 & Metoprololtartrat & $\begin{array}{l}\text { Betarezeptorenblocker } \\
\text { Antihypertonikum }\end{array}$ & $27 / 17$ & 2.547 \\
\hline 12 & Diclofenac-Na & Antirheumatikum & 5 & 2.471 \\
\hline 13 & Furosemid & Diuretikum & 36 & 2.210 \\
\hline 14 & Pentoxifyllin & Durchblutungsförderndes Mittel & 37 & 2.071 \\
\hline 15 & Verapamil-HCl & Antihypertonikum & 17 & 2.033 \\
\hline 16 & Pentaerythrithyltetranitrat ${ }^{a}$ & Koronarmittel & 55 & 1.802 \\
\hline 17 & Allopurinol & Gichtmittel & 44 & 1.695 \\
\hline 18 & Polyvidon-lod & Desinfizientium / Antiseptikum & 33 & 1.571 \\
\hline 19 & Ibuprofen & Antirheumatikum & 5 & 965 \\
\hline 20 & Glutaral $^{\mathrm{a}}$ & Desinfizientium / Antiseptikum & 33 & 951 \\
\hline 21 & Lysinamidotrizoat & $\begin{array}{l}\text { Diagnostikum } \\
\text { (Röntgenkontrastmittel) }\end{array}$ & 35 & 946 \\
\hline 22 & Acarbose & Antidiabetikum & 12 & 919 \\
\hline 23 & Roxithromycin & Antibiotikum & 10 & 915 \\
\hline 24 & Laurylpropylendiamin $^{\text {a }}$ & Desinfizientium / Antiseptikum & 33 & 905 \\
\hline 25 & Naftidrofurylhydrogenoxalat & Durchblutungsförderndes Mittel & 37 & 879 \\
\hline 26 & Torasemid-Na & Diuretikum & 36 & 837 \\
\hline 27 & Benzalkoniumchlorid $^{\text {a }}$ & Desinfizientium / Antiseptikum & 35 & 700 \\
\hline 28 & Isosorbiddinitrat & Koronarmittel & 55 & 540 \\
\hline 29 & Propranolol-HCl & $\begin{array}{l}\text { Betarezeptorenblocker } \\
\text { Antihypertonikum }\end{array}$ & $27 / 17$ & 539 \\
\hline 30 & Ampicillin & Antibiotikum & 10 & 488 \\
\hline 31 & Captopril & $\begin{array}{l}\text { Betarezeptorenblocker } \\
\text { Antihypertonikum }\end{array}$ & $27 / 17$ & 483 \\
\hline 32 & Glyoxal $^{\mathrm{a}}$ & Desinfizientium / Antiseptikum & 33 & 482 \\
\hline 33 & Formaldehyd $^{\mathrm{a}}$ & Desinfizientium / Antiseptikum & 33 & 446 \\
\hline 34 & Ciprofloxacin- $\mathrm{HCl}$ & Antibiotikum & 10 & 401 \\
\hline 35 & Talinolol & Betarezeptorenblocker & 27 & 398 \\
\hline 36 & Dimeticon & Dermatikum & 32 & 350 \\
\hline 37 & Mezlocillin-Na-Monohydrat & Antibiotikum & 10 & 325 \\
\hline 38 & Dodecylbispropylentriamin $^{a}$ & Desinfizientium / Antiseptikum & 33 & 296 \\
\hline 39 & Indometazin & Antirheumatikum & 5 & 255 \\
\hline 40 & Piperacillin-Na & Antibiotikum & 10 & 144 \\
\hline 41 & Sultamicillin & Antibiotikum & 10 & 138 \\
\hline 42 & Sulbactam-Na & Antibiotikum & 10 & 127 \\
\hline 43 & Clarithromycin & Antibiotikum & 10 & 107 \\
\hline 44 & Prednisolon & Cortikoid & 31 & 61 \\
\hline 45 & $\begin{array}{l}\text { Gestagene (Levonorgestrel, } \\
\text { Norethisteron, Norethisteronacetat) }\end{array}$ & Sexualhormone & 76 & $\begin{array}{c}50 \\
\text { (in Summe) } \\
\end{array}$ \\
\hline 46 & Prilocain- $\mathrm{HCl}$ & Lokalanästhetikum & 59 & 27 \\
\hline 47 & Lidocain- $\mathrm{HCl}$ & Lokalanästhetikum & 59 & 22 \\
\hline 48 & 5-Fluorourazil & Zytostatikum & 86 & 22 \\
\hline 49 & Cyclophosphamid & Zytostatikum & 86 & 8 \\
\hline 50 & Ifosfamid & Zytostatikum & 86 & 8 \\
\hline 51 & Medroxyprogesteronacetat & Sexualhormon / Gestagen & 76 & 4 \\
\hline 52 & Ethinylestradiol & Sexualhormon / Östrogen & 76 & 1,6 \\
\hline
\end{tabular}

${ }^{\mathrm{a}}$ :Für diese Stoffe existieren auch Anwendungen außerhalb des Regelungsbereiches des Arzneimittelgesetzes bzw. des Gesundheitswesens überhaupt. Die hier angegebenen Verbrauchsmengen berücksichtigen nur die medizinisch relevante Applikation. 
Tabelle 2: Input C: Wirkstoffauswahl aufgrund von ersten Messbefunden oberhalb der Bestimmungsgrenze in den Brandenburger Oberflächengewässern Havel (H), Teltowkanal (TK), Schwarze Elster (SE), sofern nicht schon in Input A oder B erfasst

\begin{tabular}{|c|c|c|c|c|}
\hline Lfd. Nr. & Wirkstoff & Indikation & $\begin{array}{l}\text { Indikations- } \\
\text { nummer nach } \\
\text { Rote Liste [7] }\end{array}$ & $\begin{array}{c}\text { nachgewiesen } \\
\text { in }\end{array}$ \\
\hline 53 & Phenazon & Analgetikum & 5 & $\mathrm{H}, \mathrm{TK}$ \\
\hline 54 & Naproxen & Analgetikum & 5 & $\mathrm{H}, \mathrm{TK}$ \\
\hline 55 & Propyphenazon & Analgetikum / Migränemittel & $5 / 61$ & TK \\
\hline 56 & Primidon & Antiepileptikum & 12 & TK \\
\hline 57 & Carbamazepin & Antiepileptikum & 12 & H,TK \\
\hline 58 & Bezafibrat & Lipidsenker & 58 & $\mathrm{H}, \mathrm{TK}$ \\
\hline 59 & Fenofibrinsäure & Lipidsenker-Metabolit & 58 & $\mathrm{H}, \mathrm{TK}$ \\
\hline 60 & Clofibrinsäure & Lipidsenker-Metabolit & (58) & $\mathrm{H}, \mathrm{TK}, \mathrm{SE}$ \\
\hline
\end{tabular}

Für die Wirkstoffe der Inputgruppe $\mathrm{C}$ und die vorgenannten drei Wirkstoffe aus Input A wurde als PEC-BRB für die nachfolgenden Bewertungen die maximale, bisher in einem Brandenburger Oberflächengewässer analytisch bestimmte Konzentration direkt verwendet, da entweder (wie für Stoffe aus Input C) keine Verbrauchsmengen bekannt waren oder wie bei Metoprololtartrat, Diclofenac- $\mathrm{Na}$ und Indometacin die gemessenen Konzentrationen höher waren als die abgeleiteten PEC-BRB. Derartige gegenüber der abgeleiteten PEC erhöhte Messbefunde können u.a. aus örtlichen und zeitlichen Ungleichverteilungen sowie aus jahreszeitlich veränderlichen Abbauraten in der Kläranlage [1] resultieren. Sie stellen die prinzipielle Eignung der PEC-Abschätzung nach den Gleichungen 1 und 2 nicht in Frage, wie das folgende Beispiel zeigt:

Von insgesamt 172 Messwerten für Metoprolol in repräsentativen deutschen Oberflächengewässern fanden sich vier Messwerte im Konzentrationsbereich zwischen $1 \mu \mathrm{g} / \mathrm{l}$ und $5 \mu \mathrm{g} /$, während der Median bei 0,012 $\mu \mathrm{g} / \mathrm{l}$ und das 90-Perzentil bei $0,090 \mu \mathrm{g} / \mathrm{l}$ lag [8]. Ferner variierte die Metoprolol-Konzentration an einer Brandenburger Messstelle im Verlauf von fünf Beprobungen über acht Monate zwischen < 0,06 $\mathrm{\mu g} / 1$ und $1,8 \mu \mathrm{g} / \mathrm{l}[8]$.

\subsection{Wirkungsanalyse}

Ziel der Wirkungsanalyse ist die Ermittlung der Wirkstoffkonzentration im Oberflächengewässer, unterhalb der keine Schadeffekte auf das aquatische System bzw. auf die in ihm lebenden Organismen zu erwarten sind (Predicted No Effect Concentration, PNEC).

In einem ersten Schritt erfolgte aus der im Vorfeld erstellten Sammlung ökotoxikologischer Daten die Feststellung des für jeden Wirkstoff jeweils niedrigsten Wirkungswertes für die empfindlichste Spezies. Zur Ermittlung der PNEC wurde dieser niedrigste Wirkungswert entsprechend der Gleichung (3) durch einen Sicherheitsfaktor dividiert.

PNEC

= niedrigster bekannter Wirkungswert : Sicherheitsfaktor

Sicherheitsfaktoren wurden in der Vergangenheit auch bei anderen Risikoabschätzungen für Arzneimittelwirkstoffe verwendet $[53,54]$. Sie sind notwendig, um Unsicherheiten zu berücksichtigen, die bei der Extrapolation von an einer begrenzten Anzahl von Organismen erhaltenen Testdaten auf die realen Umweltverhältnisse entstehen: Der Sicherheitsfaktor verringert sich in der Regel mit der Zunahme der Datenbasis und der Aussagefähigkeit der durchgeführten Tests. Daten aus Langzeittests besitzen verglichen mit Akuttests dabei generell eine größere Aussagekraft, so dass der Sicherheitsfaktor bei Vorliegen derartiger Daten entsprechend verringert werden kann.

Die in dieser Arbeit zur Ermittlung der PNEC verwendeten Sicherheitsfaktoren sind in Tabelle 4 dargestellt. Die erste Spalte der nachfolgenden Tabelle gibt Auskunft über die Anzahl der Trophiestufen des aquatischen Ökosystems (z.B. Algen als Primärproduzenten, Daphnien als Primärkonsumenten, Fische als Sekundärkonsumenten und Bakterien als Destruenten), für die ökotoxikologische Wirkungsdaten zur Verfügung standen.

Ausgangspunkt bei der Ableitung der Sicherheitsfaktoren war der im EMEA-Papier [10] vorgeschlagene Wert von 1000 auf die niedrigste $\mathrm{EC}_{50}$ für drei trophische Stufen des aquatischen Ökosystems. Im Unterschied zur üblichen Chemikalienbewertung nach TGD, bei der in der Regel für alle trophischen Stufen mindestens akute Toxizitätsdaten vorliegen, fehlen derzeit für die Bewertung der Humanarzneimittel teilweise Daten zur akuten Toxizität für eine oder auch mehrere Trophieebenen, während Daten aus Tests zur chronischen Toxizität nahezu ganz fehlen. Um dennoch eine erste und orientierende Risikobewertung, die alle wichtigen trophischen Stufen einschließt, mit einem geschlossenen Bewertungsansatz vornehmen zu können, haben wir, ausgehend vom Wert 1000 , in beide Richtungen fehlendes oder zusätzliches Wissen formal mit einer Erhöhung oder Verringerung des Sicherheitsfaktors berücksichtigt. Dabei haben wir für jede fehlende (zusätzliche) Trophiestufe den Sicherheitsfaktor um den Faktor 5 erhöht (verringert). Diesen Faktor 5 haben wir gewählt, weil die so entstandene Spannweite der Sicherheitsfaktoren zwischen 200 (bei vorhandenen Wirkungswerten für 4 trophische Stufen) und 25000 (bei vorhandenen Wirkungswerten für nur eine trophische Stufe) in grober Näherung auch die mittlere Spannweite der verschiedenen Akuttoxizitäten $\left(\mathrm{EC}_{50} / \mathrm{LC}_{50}\right)$ zwischen empfindlichster und unempfindlichster Trophiestufe widerspiegelt (Tabelle 5, Beispiel Ciprofloxacin-HCl für eine höhere Spannweite, Paracetamol für eine mittlere Spannweite und Clofibrinsäure für eine niedrigere Spannweite der $\mathrm{EC}_{50} / \mathrm{LC}_{50}$ ). 
Tabelle 3: Ableitung einer PEC-BRB für das Land Brandenburg für Stoffe nach Input $A$ und $B$

\begin{tabular}{|c|c|c|c|c|c|c|}
\hline $\begin{array}{l}\text { Lfd. } \\
\text { Nr. }\end{array}$ & Wirkstoff & $\begin{array}{c}\text { Jährliche } \\
\text { Verbrauchsmenge } \\
\text { in BRB in kg [5] }\end{array}$ & $\begin{array}{l}\mathrm{PEC}_{\mathrm{wc}}-\mathrm{BRB} \\
\text { in } \mu \mathrm{g} / \mathrm{l}\end{array}$ & $\begin{array}{c}\text { ausgeschiedener } \\
\text { Wirkstoffanteil in \% } \\
\text { nach Passage des } \\
\text { menschlichen } \\
\text { Organismus [34] }\end{array}$ & $\begin{array}{c}\text { Rückhaltung in der } \\
\text { Kläranlage in \% [34] } \\
A=\text { Abbau } \\
S=\text { Sorption } \\
E=\text { Eliminierung }\end{array}$ & $\begin{array}{l}\text { PEC-BRB } \\
\text { in } \mu g / 1\end{array}$ \\
\hline 1 & Acetylsalicylsäure & 24.269 & 17,05 & $5-10^{b}$ & $81, \mathrm{E}$ & 0,32 \\
\hline 2 & Acetylcystein & 10.561 & 7,42 & $<10^{\mathrm{c}}$ & $>0$, leichter $\mathrm{A}^{\mathrm{C}}$ & $<0,74$ \\
\hline 3 & Phenoxypropanol-Isomere & 7.892 & 5,54 & 100 (keine Passage) & 24-88, A & 4,21 \\
\hline 4 & Metformin- $\mathrm{HCl}$ & 7.620 & 5,35 & 100 & $0, \mathrm{~A}$ & 5,35 \\
\hline 5 & Paracetamol & 6.753 & 4,74 & $5+90 \%$ Glucuronid) & $>99, \mathrm{E}$ & $<0,045$ \\
\hline 6 & Glucoprotamin & 5.023 & 3,53 & 100 (keine Passage) & 58-94 & 1,48 \\
\hline 7 & \begin{tabular}{|l|} 
Iodixanol \\
\end{tabular} & 4.940 & 3,47 & 100 & $0, \mathrm{E}$ & 3,47 \\
\hline 8 & Metamizol-Na & 4.649 & 3,27 & $<10$ & $20-50$ & 0,26 \\
\hline 9 & \begin{tabular}{|l|}
$\begin{array}{l}\text { Cocospropylendiamin- } \\
\text { guaniacetat }\end{array}$ \\
\end{tabular} & 3.157 & 2,22 & 100 (keine Passage) & $0^{c}$ & 2,22 \\
\hline 10 & \begin{tabular}{|l} 
Theophyllin \\
\end{tabular} & 2.790 & 1,96 & $7-13$ & $>0$, leichter $\mathrm{A}$ & $<0,25$ \\
\hline 11 & Metoprololtartrat & 2.547 & 1,79 & $3-10$ & $67, \mathrm{E}$ & $0,059 / 1,8^{d}$ \\
\hline 12 & Diclofenac-Na & 2.471 & 1,74 & 15 & $31-69, E, 4, S$ & $0,18 / 0,47^{d}$ \\
\hline 13 & \begin{tabular}{|l} 
Furosemid \\
\end{tabular} & 2.210 & 1,55 & 90 & $0^{c}$ & 1,4 \\
\hline 14 & \begin{tabular}{|l|} 
Pentoxifyllin \\
\end{tabular} & 2.071 & 1,45 & $<5$ & $>80, A, 27 \mathrm{~S}$ & $<0,015$ \\
\hline 15 & Verapamil-HCl & 2.033 & 1,43 & 20 & $0^{c}$ & 0,29 \\
\hline 16 & Pentaerythrithyltetranitrat & 1.802 & 1,27 & $<30$ & $0^{c}$ & 0,41 \\
\hline 17 & Allopurinol & 1.695 & 1,19 & 27 & $0^{\mathrm{c}}$ & 0,32 \\
\hline 18 & Polyvidon-lod & 1.571 & 1,10 & 100 (keine Passage) & $0-<20, A$ & 1,10 \\
\hline 19 & \begin{tabular}{|l|} 
Ibuprofen \\
\end{tabular} & 965 & 0,68 & 15 & $58-90, \mathrm{E}$ & 0,042 \\
\hline 20 & Glutaral & 951 & 0,67 & 100 (keine Passage) & $74-100, A$ & 0,17 \\
\hline 21 & Lysinamidotrizoat & 946 & 0,66 & $100^{\mathrm{c}}$ & $0^{c}$ & 0,66 \\
\hline 22 & Acarbose & 919 & 0,65 & 53 & $47, \mathrm{~A}$ & 0,18 \\
\hline 23 & Roxithromycin & 915 & 0.64 & $50^{\mathrm{c}}$ & $30-50, E$ & 0,22 \\
\hline 24 & Laurylpropylendiamin & 905 & 0,64 & 100 (keine Passage) & $>90, \mathrm{~A}$ & 0,064 \\
\hline 25 & Naftidrofurylhydrogenoxalat & 879 & 0,62 & 0,5 & $0, \mathrm{~A}$ & 0,003 \\
\hline 26 & \begin{tabular}{|l|} 
Torasemid-Na \\
\end{tabular} & 837 & 0,59 & 24 & $0^{c}$ & 0,14 \\
\hline 27 & Benzalkoniumchlorid & 700 & 0,49 & 100 (keine Passage) & $36-100, A$ & 0,31 \\
\hline 28 & Isosorbitdinitrat & 540 & 0,38 & $<5$ & $0^{c}$ & 0,019 \\
\hline 29 & Propranolol-HCl & 539 & 0,38 & $1-4$ & $95, \mathrm{E}$ & 0,0008 \\
\hline 30 & Ampicillin & 488 & 0,34 & 90 & $48, A$ & 0,16 \\
\hline 31 & Captopril & 483 & 0,34 & $40-50$ & $0^{c}$ & 0,17 \\
\hline 32 & Glyoxal & 482 & 0,34 & 100 (keine Passage) & $>90, \mathrm{~A}$ & 0,034 \\
\hline 33 & \begin{tabular}{|l|} 
Formaldehyd \\
\end{tabular} & 446 & 0,31 & 100 (keine Passage) & $63->90, A$ & 0,11 \\
\hline 34 & Ciprofloxacin- $-\mathrm{HCl}$ & 401 & 0,31 & 70 & $0, \mathrm{~A}$ & 0,22 \\
\hline 35 & Talinolol & 398 & 0,28 & 99 & $0^{c}$ & 0,277 \\
\hline 36 & Dimeticon & 350 & 0,25 & 100 (orale Applikation) & $0^{\mathrm{c}}$ & 0,25 \\
\hline 37 & Mezlocillin-Na-Monohydrat & 325 & 0,23 & 100 & $0^{c}$ & 0,23 \\
\hline 38 & Dodecylbispropylentriamin & 296 & 0,21 & 100 (keine Passage) & $91-96, \mathrm{~A}$ & 0,019 \\
\hline 39 & Indometazin & 255 & 0,18 & $10-20$ & $38-83, \mathrm{E}$ & $0,022 / 0,026^{d}$ \\
\hline 40 & Piperacillin-Na & 144 & 0,10 & $60-80$ & $0^{c}$ & 0,08 \\
\hline 41 & Sultamicillin & 138 & 0,10 & $42-86$ & $0^{c}$ & 0,086 \\
\hline 42 & Sulbactam-Na & 127 & 0,09 & $75-80$ & $0^{c}$ & 0,072 \\
\hline 43 & Clarithromycin & 107 & 0,08 & $20-30$ & $61, \mathrm{E}$ & 0,009 \\
\hline 44 & Prednisolon & 61 & 0,04 & $<10^{c}$ & $0^{c}$ & 0,004 \\
\hline 45 & $\begin{array}{l}\text { Kombiwirkstoff } \\
\text { (Levonorgestrel, } \\
\text { Norethisteron, } \\
\text { Norethisteronacetat) }\end{array}$ & $\begin{array}{c}\Sigma 50 \text { Anteil zu je } 1 / 3 \\
\text { angegenommen }\end{array}$ & $\begin{array}{l}0,03 \\
0,01 \\
0,01 \\
0,01\end{array}$ & $\begin{array}{l}100^{\mathrm{c}} \\
10^{\mathrm{c}} \\
3,1-8\end{array}$ & $\begin{array}{c}70, A \\
0^{c} \\
72-100, A\end{array}$ & $\begin{array}{c}0,003 \\
0,001 \\
0,00022 \\
\end{array}$ \\
\hline 46 & Prilocain- $\mathrm{HCl}$ & 27 & 0,02 & $100^{c}$ & $0^{c}$ & 0,02 \\
\hline 47 & \begin{tabular}{|l|} 
Lidocain- $\mathrm{HCl}$ \\
\end{tabular} & 22 & 0,02 & $5-10$ & $0^{c}$ & 0,002 \\
\hline 48 & \begin{tabular}{|l|} 
5-Fluorourazil \\
\end{tabular} & 22 & 0,02 & 15 & $0, \mathrm{~A}$ & 0,003 \\
\hline 49 & Cyclophosphamid & 8 & 0,005 & $10-40$ & $0, \mathrm{E}, 0 \mathrm{~A}$ & 0,002 \\
\hline 50 & Ifosfamid & 8 & 0,005 & $15-53$ & $0, \mathrm{~A}$ & 0,0027 \\
\hline 51 & \begin{tabular}{|l|} 
Medroxyprogesteronacetat \\
\end{tabular} & 4 & 0,003 & 25-60 (Konjugate) & $0^{c}$ & 0,0018 \\
\hline 52 & Ethinylestradiol & 1,6 & 0,001 & 100 (Konjugate) & $\sim 0, \mathrm{E}, 0 \mathrm{~A}$ & 0,001 \\
\hline
\end{tabular}


Tabelle 4: Sicherheitsfaktoren zur Ermittlung der PNEC

\begin{tabular}{l|c|c}
\hline $\begin{array}{l}\text { Ökotoxikologische Wirkungsdaten } \\
\text { vorhanden für: }\end{array}$ & $\begin{array}{c}\text { Wirkkonzentration als NOEC bzw. } \\
\text { als } \mathrm{EC}_{0}-\mathrm{EC}_{10}\end{array}$ & Wirkkonzentration als $\mathrm{EC}_{50}$ \\
\hline 4 Trophiestufen & 100 & 200 \\
\hline 3 Trophiestufen & 500 & 1000 \\
\hline 2 Trophiestufen & 2500 & 5000 \\
\hline 1 Trophiestufe & 12500 & 25000 \\
\hline
\end{tabular}

Tabelle 5: Vergleich der $\mathrm{E}(\mathrm{L}) \mathrm{C}_{50}$ von Ciprofloxacin- $\mathrm{HCl}$, Clofibrinsäure und Paracetamol für verschiedene Trophiestufen, (Angaben in mg/l)

\begin{tabular}{|c|c|c|c|c|}
\hline Wirkstoff & Algen & Krebse & Fische & Bakterien \\
\hline Ciprofloxacin- $\mathrm{HCl}$ & $\begin{array}{l}33 \\
\text { Sc. subspicatus } \\
\mathrm{EC}_{50}, 72 \mathrm{~h} \\
\text { Hemmung } \\
\text { Zellvermehrung [18] }\end{array}$ & $\begin{array}{l}176 \\
\text { Daphnien } \\
\text { (nicht näher bezeichnet } \mathrm{EC}_{50} \text { ) [18] }\end{array}$ & $\begin{array}{l}1000 \\
\text { Brachydanio rerio } \\
\mathrm{LC}_{50}, 96 \mathrm{~h}[18]\end{array}$ & $\begin{array}{l}0,0093 \\
\text { Ps. putida } \\
\text { EC }_{50}[18]\end{array}$ \\
\hline Clofibrinsäure & $\begin{array}{l}145 \\
\text { Desmodesmus. subspicatus } \\
\mathrm{EC}_{50} \text {, Wachstum [57] }\end{array}$ & $\begin{array}{l}>200 \\
\text { C. dubia } \\
48 \mathrm{~h}, \mathrm{EC}_{50}[56] \\
75 \\
\text { Daphnia magna } \\
\mathrm{EC}_{50}[57]\end{array}$ & $\begin{array}{l}86 \text { / } 126 \\
\text { Fischembryonen, } \\
\text { EC }_{50} \\
\text { Sterblichkeit / Pulsrate } \\
\text { (Test n. Schulte und Nagel) [1] }\end{array}$ & $\begin{array}{l}91,8 \\
\text { Vibrio fischeri } \\
\mathrm{EC}_{50} \\
\text { Microtox } 30 \mathrm{~min} \\
{[56]}\end{array}$ \\
\hline Paracetamol & $\begin{array}{l}134 \\
\text { nicht näher bezeichnet } \\
\mathrm{EC}_{50}[1]\end{array}$ & $\begin{array}{l}\mathbf{9 , 2 - 5 5 , 5} \\
\text { Daphnia magna } \\
\mathrm{EC}_{50}, 48 \text { und } 24 \mathrm{~h}[23,27]\end{array}$ & $\begin{array}{l}378 \text { / } 920 \\
\text { Fischembryonen, } \mathrm{EC}_{50} \\
\text { Sterblichkeit / Pulsrate } \\
\text { (Test n. Schulte und Nagel) [1] }\end{array}$ & $\begin{array}{l}650 \\
\text { nicht näher bezeichnet } \\
\mathrm{EC}_{50} \\
\text { Lumineszenzhemmung [1] }\end{array}$ \\
\hline
\end{tabular}

Dieses formale Vorgehen hat den Nachteil, dass Stoffe, deren Akuttoxizitäten zwischen den Trophiestufen in Wirklichkeit wenig schwanken (wie z.B. Clofibrinsäure), bei Fehlen von Daten für einzelne trophische Stufen zu streng bewertet werden. In der Realität vorhandene große Schwankungsbreiten der $\mathrm{EC}_{50}$ l $\mathrm{LC}_{50}$ (wie z.B. Ciprofloxacin-HCl) könnten bei dieser Vorgehensweise das Umweltrisiko u.U. aber unterschätzen. Dieser Faktor 5 sollte deshalb als ein Kompromiss angesehen werden, der für eine grobe Erstbewertung der Stoffe die derzeitige schlechte und heterogene Datenlage auzugleichen versucht.

Andererseits wurde mit dem Faktor 2 berücksichtigt, ob es sich bei der niedrigsten Wirkkonzentration um eine $\mathrm{EC}_{50}$ oder um eine NOEC bzw. $\mathrm{EC}_{0}-\mathrm{EC}_{10}$ handelt. Hinweise für diese Annahme gibt der Verlauf einzelner zur Verfügung stehender Konzentrations-Wirkungs-Beziehungen (z.B. Fischund Daphnientoxizität von Diclofenac-Na [26] oder Ciprofloxacin-HCl [18]), wobei jedoch auch in diesem Punkt vom Vorhandensein von stoff- und wirkungsspezifischen Variationen auszugehen ist.

\subsection{Bewertung der Umweltrelevanz}

Die Vorgehensweise zur Bewertung der Umweltrelevanz der ausgewählten Arzneimittelwirkstoffe, für die ökotoxikologische Wirkungsdaten vorhanden sind, unterscheidet sich grundsätzlich von jener für Wirkstoffe ohne verfügbare ökotoxikologische Wirkungsdaten.

Konnte bei vorhandenen Wirkungsdaten entsprechend 1.3 eine PNEC berechnet werden, so wurde zunächst das Verhältnis von PEC-BRB : PNEC, also von geschätzter oder gemessener Konzentration im Oberflächenwasser zu der Wirkstoffkonzentration, bei der nach dem jetzigen Stand der Erkenntnis keine Schadwirkungen auf das aquatische System zu erwarten sind, bestimmt.

Ist dieses Verhältnis $<1$ und liegen keine anderen Hinweise für eine Umweltrelevanz (z.B. Akkumulationspotential, Persistenz, siehe unten) vor, so wird nach jetzigem Kenntnisstand für diesen Wirkstoff nicht vom Vorhandensein einer Umweltrelevanz ausgegangen.

Ist das Verhältnis PEC-BRB : PNEC $\sim 1$ oder $>1$, so ist unabhängig von den o.g. sonstigen Parametern zum Umweltverhalten vom Vorhandensein einer Umweltrelevanz bzw. einer hohen Umweltrelevanz ( $a b$ PEC-BRB : PNEC > 10) auszugehen.

Bei fehlenden ökotoxikologischen Wirkungsdaten ist eine konsistente Risikobewertung nicht möglich. In Abhängigkeit von der Verfügbarkeit weiterer Daten erfolgte aber aufgrund chemisch-physikalischer Stoffdaten, Erkenntnissen zur Persistenz bzw. zum Akkumulationspotential des Stoffes in der Unwelt, aber auch aufgrund nachgewiesener kanzerogener, mutagener, reproduktionstoxischer oder endokriner Wirkungen auf den Human- bzw. Säugerorganismus eine vorläufige Beurteilung der Umweltrelevanz.

Als Hinweis für eine Umweltrelevanz im aquatischen Ökosystem außerhalb des PEC-BRB : PNEC-Kriteriums gilt, wenn: a: der Oktanol/Wasser-Verteilungskoeffizient $>1000$ (bzw. $\log K_{\text {ow }}>3$ ) ist oder ein Biokonzentrationsfaktor (BCF) von $>100$ für einen aquatischen Organismus vorliegt und somit eine beachtliche Bioakkumulation möglich ist, oder 
b: die Halbwertszeit des Wirkstoffes im Oberflächenwasser mehr als 2 Monate beträgt (Screening - Kriterium des Anhangs D der POP-Konvention [14]), oder

c: Tests der biologischen Abbaubarkeit Abbauraten von weniger als $90 \%$ aufweisen, oder

d: Untersuchungen zur Kläranlageneliminierung (z.B. Ermittlung der Konzentrationsdifferenz zwischen Kläranlagenzulauf und Kläranlagenablauf) Eliminationsraten von weniger als $30 \%$ ergeben oder

e: es für den Human- oder Säugerorganismus Hinweise auf kanzerogene und/oder mutagene und/oder reproduktionstoxische und/oder endokrine Wirkungen gibt.

Wirkstoffe, die zwei oder mehr der Kriterien a-e erfüllen, sind aus Tabelle 8 in 2.3 ersichtlich.

Um die Datenbasis zur Beurteilung des Umweltverhaltens von Arzneimittelwirkstoffen zu erweitern, wurden mit Hilfe von quantitativen Struktur-Wirkungs-Beziehungen (Quantitative Structure Activity Relationship, QSAR), für 57 Wirkstoffe Oktanol/Wasser-Verteilungskoeffizienten ${ }^{1}$, Biokonzentrationsfaktoren (BCF $=\log K_{\text {ow }}-1,3$ ), Wasserlöslichkeiten ${ }^{2}$, HenryKonstanten ${ }^{2}$, Dampfdrücke ${ }^{3}$ sowie Mindestfischtoxizitäten ${ }^{4}$ berechnet. Desweiteren wurden aus den so ermittelten chemisch-physikalischen Daten Verteilungstendenzen für die Wirkstoffe in den verschiedenen Umweltkompartimenten für wasserreiche und wasserarme Szenarien abgeleitet (Level-I-Verteilungen nach Mackay [11,12]).

Der Oktanol / Wasser-Verteilungskoeffizient kennzeichnet das Konzentrationsverhältnis eines Stoffes in den zwei nicht mischbaren Flüssigkeiten n-Oktanol und Wasser im Gleichgewichtszustand. Aus diesem Verteihungskoeffizienten lässt sich durch die o.g. Beziehung in einfacher Art und Weise ein Biokonzentrationsfaktor als ein Maß für die direkte Aufnahme eines Stoffes in den aquatischen Organismus aus der Umgebung abschätzen. Aus der Henry-Konstante und dem Dampfdruck können dagegen Aussagen zur Tendenz des Stoffübergangs in das Kompartiment Luft abgeleitet werden.

Unter der Mindestfischtoxizität ist eine mit der Lipophilie eines Stoffes korrelierende Basistoxizität gegenüber Guppys, die aufgrund von narkotischen Wirkungen zu erwarten ist, zu verstehen. Die Berechnung dieser Mindestfischtoxizität erfolgte nach der Könemann-Gleichung (siehe [13]): $\log 1 /$ $\mathrm{LC}_{50}[\mathrm{~mol} / \mathrm{l}]=0,871 * \log K_{\text {ow }}+1,13$.

Bei den Berechnungen der Level-I-Verteilungen nach Mackay handelt es sich um eine grobe Näherung mit folgender stark vereinfachter Annahme: Es existiert ein thermodynamisches Gleichgewicht zwischen allen Kompartimenten ohne weiteren Input bzw. Output. Zur Berechnung für die Szenarien wasserreiche Region und wasserarme Region wurden die in [11] genannten Parameter angewendet. Beispielsweise wurde für eine wasserreiche Region eine 30\%-ige Wasserbedeckung, für die wasserarme Region dagegen lediglich eine 2,5\%-ige Wasserbedeckung der Oberfläche (repräsentativ für die Region Leipzig-Halle, aber auch für das Land Bran-

\footnotetext{
1 Daylight, KOWIN

2 nach Howard

${ }^{3}$ Antoine-Gleichung

4 Könemann-Gleichung
}

denburg) gewählt [11]. Im Rahmen der Arzneimittelproblematik können Fragen der Kompartimentverteilungen beispielsweise bei der Beantwortung der Frage von Bedeutung sein, inwieweit ein Wirkstoff während der Kläranlagenpassage zur Anreicherung im Klärschlamm tendiert.

Die mit Hilfe von QSAR-Betrachtungen abgeleiteten Werte sind, sofern für die jeweiligen Parameter keine experimentell bestimmten Werte zur Verfügung standen, in den Datenblättern in [34] zusammengestellt. In diesem Aufsatz sollen im Ergebnisteil (siehe 2.3) beispielhaft lediglich die berech-' neten minimalen Fischtoxizitäten im Vergleich mit experimentell bestimmten Fischtoxizitäten diskutiert werden.

\subsection{Empfehlungskriterien zur Analytik}

Die zur Analytik vorgeschlagenen Wirkstoffe wurden entsprechend der für sie festgestellten Umweltrelevanz in vier Prioritätengruppen eingeteilt.

Stoffe mit einer nach dem PEC-BRB : PNEC-Verhältnis festgestellten ökotoxikologischen Relevanz (PEC-BRB : PNECVerhältnis von $\sim 1$ oder $>1$ ) sollten mit höchster Priorität (Priorität A) routinemäßig in Brandenburger Oberflächengewässern überwacht werden.

Wirkstoffe, die bei ausschließlicher Betrachtung des PECBRB : PNEC-Verhältnisses zwar nicht auf eine wirkungsseitig belegbare Umweltrelevanz schließen lassen, jedoch mindestens zwei Hinweiskriterien nach 1.4 erfüllen, werden in der Prioritätengruppe $B$ für eine zunächst stichprobenartige Analytik z.B. in Oberflächengewässern mit hohem Abwasseranteil empfohlen. Diesen Stoffen werden hinsichtlich der Analysenpriorität jene Wirkstoffe gleichgestellt, für welche die Bestimmung des PEC-BRB : PNEC-Verhältnisses mangels geeigneter ökotoxikologischer Wirkungsdaten derzeit nicht möglich ist, die aber dennoch mindestens zwei Hinweiskriterien für eine Umweltrelevanz nach 1.4 erfüllen.

Wirkstoffe, für die wegen fehlender Daten weder eine Bewertung der Umweltrelevanz nach dem PEC-BRB : PNEC-Konzept noch eine Prognose des Umweltverhaltens entsprechend den Kriterien nach 1.4 möglich ist, bilden die Prioritätengruppe C. Für diese Stoffe ist durch Erweiterung der ökotoxikologischen Datenbasis ein mögliches Umweltgefährdungspotential schnellstmöglich aufzuklären. Bis dahin sollten sie im Rahmen eines erweiterten Analysenprogramms an wenigen repräsentativen Probenahmestellen in einem größeren Zeitraster analysiert werden.

Für Wirkstoffe mit einem Verhältnis PEC-BRB : PNEC $<1$ und ohne Anhaltspunkte für eine Umweltrelevanz gemäß den Kriterien nach 1.4 wird bis zum Vorliegen anders lautender ökotoxikologischer Erkenntnisse eine analytische Überwachung nicht als notwendig erachtet (Analysenpriorität 0).

\section{Ergebnisse}

Gemäß der Vorgehensweise bei der Bewertungsmethodik soll auch die Ergebnisbetrachtung separat für die Expositionsanalyse, die Wirkungsanalyse und die Bewertung der Umweltrelevanz erfolgen. 


\subsection{Expositionsanalyse}

In Abb. 2 sind die nach der in 1.2 vorgestellten Methodik für Brandenburger Oberflächengewässer abgeleiteten Wirkstoffkonzentrationen, sofern sie einen Wert von $0,1 \mathrm{\mu g} / \mathrm{l}$ überschreiten, dargestellt. Bei den grau unterlegten Konzentrationsangaben handelt es sich um die Maxima von analytisch in Brandenburger Oberflächengewässern ermittelten Messwerten $[8,9]$.

Festzustellen ist zunächst, dass von den betrachteten $60 \mathrm{Arz}-$ neimittelwirkstoffen 8 (1 Antidiabetikum, 4 Desinfektionsmittel, 1 Röntgenkontrastmittel, 1 Betarezeptorenblocker und 1 Diuretikum) im Brandenburger Oberflächenwasser in einer Konzentration $>1 \mu \mathrm{g} / \mathrm{l}$ zu erwarten sind. Dabei wurde für das Antidiabetikum Metformin-HCl mit $5,35 \mu \mathrm{g} / \mathrm{l}$ die höchste PEC-BRB von allen betrachteten Wirkstoffen ermittelt. Für weitere 27 Arzneimittelwirkstoffe wird eine PECBRB zwischen $1 \mu \mathrm{g} / \mathrm{l}$ und $0,1 \mu \mathrm{g} / \mathrm{l}$ erwartet. In dieser Gruppe finden sich u.a. neben den häufig gebrauchten Schmerz- und Rheumamitteln (Diclofenac-Na, Phenazon, Acetylsalicylsäure, Metamizol-Na) auch einige Antibiotika (Roxithromycin, Ciprofloxacin-HCl, Mezlocillin-Na-Monohydrat) und die Antiepileptika Carbamazepin und Primidon. Für alle weiteren Wirkstoffe ist eine PEC-BRB von $<0,1 \mu \mathrm{g} / \mathrm{l}$ zu erwarten.

Betrachtet man die drei bei der Ermittlung der PEC-BRB berücksichtigten Einflussfaktoren jährliche Verbrauchsmenge, Humanmetabolismus und Kläranlageneliminierung (Abb. 3), so ist ein wesentlicher Einfluss der jährlichen Verbrauchsmenge eines Wirkstoffes auf die PEC-BRB nur dann festzustellen, wenn der Humanmetabolismus und/oder die Kläranlageneliminierung wenig zur Verringerung der Wirkstoffmenge auf dem Weg ins Oberflächengewässer beitragen. Dies trifft insbesondere für den Wirkstoff Metformin- $\mathrm{HCl} \mathrm{zu,}$ dessen relativ große jährliche Verbrauchsmenge bei nahezu fehlender Humanmetabolisierung und Kläranlageneliminierung unmittelbar einen Spitzenplatz in der PEC-BRB-Rangliste bewirkt. Ähnlich inert wie Metformin- $\mathrm{HCl}$ verhält sich sowohl hinsichtlich des Humanmetabolismus als auch bezüglich der Kläranlageneliminierung das Röntgenkontrastmittel Iodixanol. Ganz im Gegensatz dazu erfolgt bei der Acetylsalicylsäure eine weitgehende Metabolisierung im menschlichen Organismus sowie eine umfangreiche Kläranlageneliminierung. Beide genannten Faktoren bewirken, dass trotz sehr hoher Verbrauchsmengen die geschätzte Konzentration an unveränderter Acetylsalicylsäure im Oberflächengewässer als eher gering anzusehen ist. Beim Glucoprotamin findet, wie bei nahezu allen Desinfektionsmitteln, durch eine fehlende Passage des Organismus kein Humanmetałolismus statt, allerdings kommt es in der Kläranlage zu einem merklichen Abbau [15]. Ebenso wird Paracetamol in der Kläranlage nahezu vollständig eliminiert, nach dem die als Folge der Biotransformation im menschlichen Organismus gebildeten Glucuronide teilweise instabil sind und später wieder gespalten werden [16]. Bei vielen Antibiotika gelangen, wie hier für Ciprofloxacin- $\mathrm{HCl}$ und Ampicillin beispielhaft gezeigt, wesentliche Anteile der ursprünglich verabreichten Menge unverändert in das Oberflächenwasser.

Eine Verifizierung der abgeschätzten PEC-BRB ist aufgrund von fehlenden Analysenwerten für Brandenburger Ober- flächengewässer derzeit für die meisten Wirkstoffe nicht möglich. Für die Mehrzahl der Wirkstoffe, für die sowohl PECAbschätzungen als auch Messwerte [8] vorliegen, lässt sich eine gute Übereinstimmung zwischen Modell und Realität feststellen. Ausnahmen sind beispielhaft in 1.2 diskutiert.

\subsection{Wirkungsanalyse}

Zur Vorhersage einer Wirkstoffkonzentration, unterhalb der keine schädigenden Effekte auf das aquatische System zu erwarten sind (PNEC), sind zunächst ökotoxikologische Wirkungswerte für die verschiedenen Arzneimittelwirkstoffe erforderlich. Weil derartige ökotoxikologische Prüfungen bislang im Zulassungsverfahren für Humanarzneimittelwirkstoffe nicht gefordert wurden, ist die Datenlage im Vergleich der verschiedenen Wirkstoffe sehr unterschiedlich, insgesamt aber als dürftig zu bezeichnen.

Für insgesamt 40 der untersuchten 60 Wirkstoffe bzw. Wirkstoffmetaboliten konnte mindestens ein ökotoxikologischer Wirkungswert ermittelt werden. Viele der Wirkdaten sind den Sicherheitsdatenblättern für die Wirkstoffe entnommen und nicht in jedem Fall validiert. Die hinsichtlich der verschiedenen Wirkstoffe unterschiedliche Datenlage und qualität wird bei der Ermittlung der PNEC durch unterschiedlich hohe Sicherheitsfaktoren ausgeglichen (siehe 1.3). Die Ableitung einer PNEC bei Vorliegen lediglich eines ökotoxikologischen Wirkungswertes (eine Trophiestufe) ist trotz der hohen Sicherheitsfaktoren sehr unsicher und nur mit der ohnehin schlechten Datenlage und dem Ziel-der Vorgabe erster Trends zur Bewertung des Umweltrisikos überhaupt zu rechtfertigen. Allgemein gilt, je schlechter die Basis ökotoxikologischer Wirkungsdaten ist, desto unsicherer ist die abgeleitete PNEC und das sich vielleicht aus ihr ergebende Umweltrisiko, und desto notwendiger sind künftige, möglicherweise anders lautende Neubewertungen.

Tabelle 6 gibt eine Übersicht über die zur Ableitung der PNEC verwendeten jeweils niedrigsten ökotoxikologischen Wirkungswerte und die zur Anwendung gelangten Sicherheitsfaktoren.

Betrachtet man exemplarisch die Wirkstoffe der ersten zwei Gruppen aus Tabelle 6 mit einer hohen Toxizität gegenüber Wasserorganismen (PNEC $<1 \mu \mathrm{g} / \mathrm{l}$ ), so finden sich in dieser Gruppe neben dem Sexualhormon Ethinylestradiol auch das Antibiotikum Ciprofloxacin-HCl, das Antiepileptikum Carbamazepin, der Lipidsenkermetabolit Clofibrinsäure und die Desinfektionsmittelwirkstoffe Benzalkoniumchlorid und Glucoprotamin. Dabei stellen im Falle der Clofibrinsäure, des Carbamazepins und beim Glucoprotamin die Daphnien, beim Benzalkoniumchlorid die Algen und beim Ciprofloxacin$\mathrm{HCl}$ die Bakterien die empfindlichsten Organismen im aquatischen System dar. Die extrem niedrige PNEC für Ethinylestradiol resultiert aus der im unteren ng/-Bereich einsetzenden unphysiologischen Induktion der Vitellogeninsynthese bei Fischen [48-50] sowie aus der ebenfalls in diesem Konzentrationsbereich beginnenden negativen Beeinflussung von Wachstum, Überlebensrate und Reproduktion (einschließlich der Verschiebung des Geschlechterverhälmisses) bei Pimephales promelas [52]. Da es sich hier um spezifische Wirkungen handelt, muss davon ausgegangen werden, dass durch die bisher 


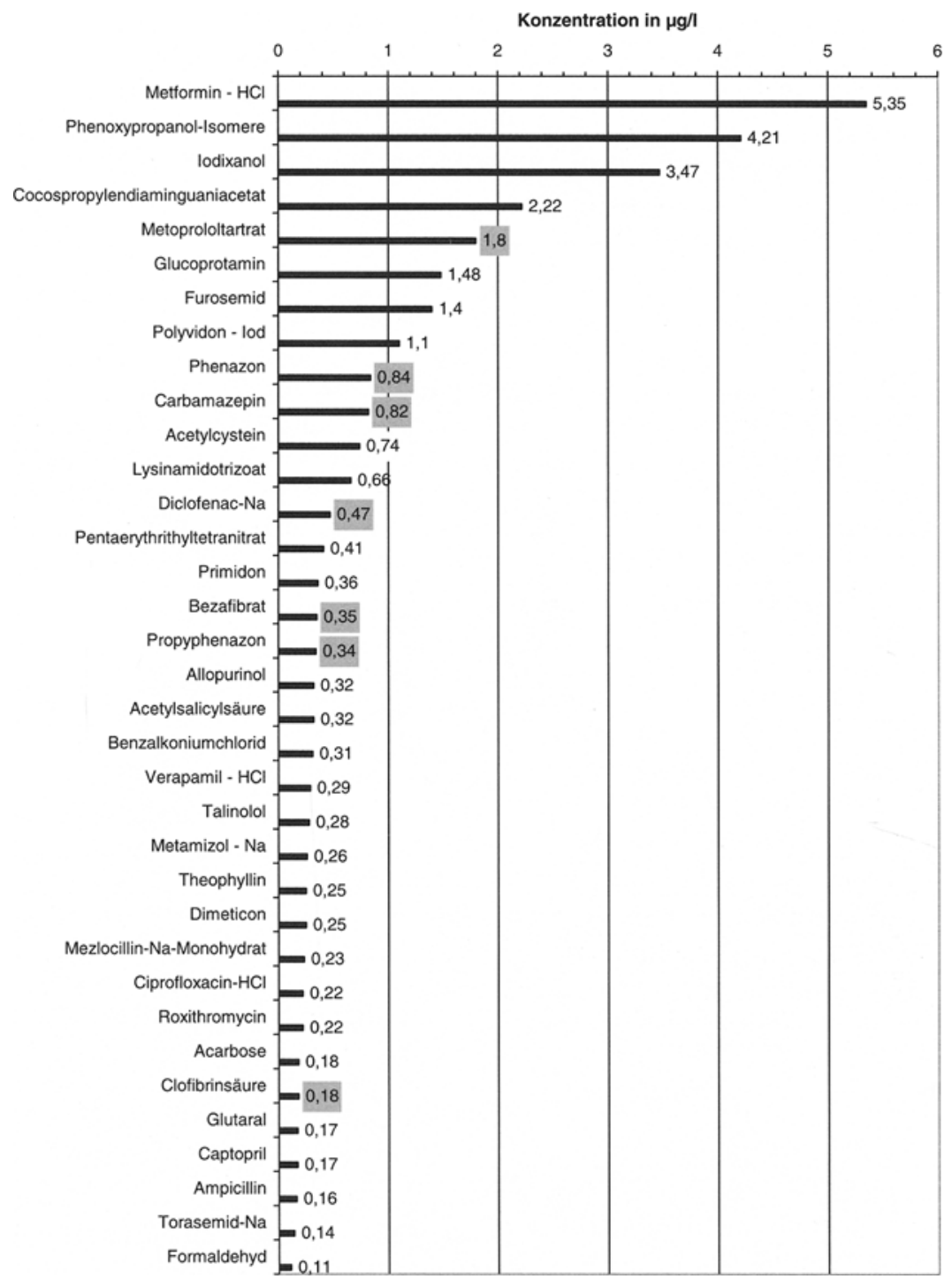

Abb. 2: PEC-BRB für Arzneimittel in Brandenburger Oberflächengewässern (für Wirkstoffe mit einer $P E C>0,1 \mu g / l)$. Bei den grau unterlegten Konzentrationswerten handelt es sich um die Maxima realer Messwerte in Brandenburger Gewässern

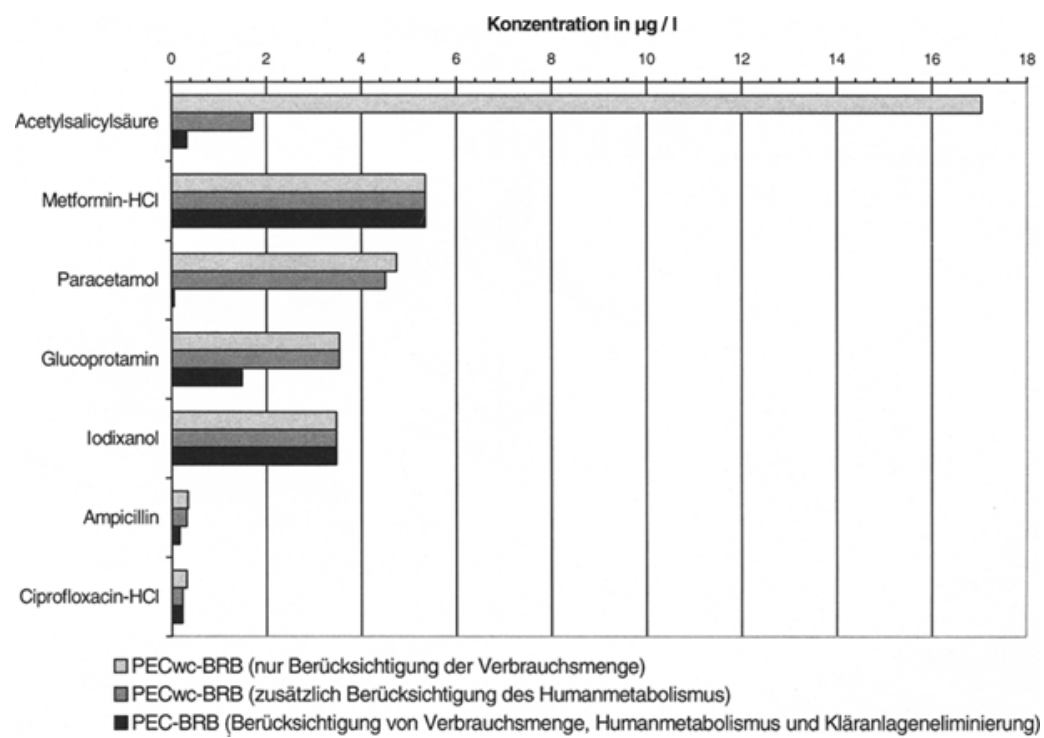

Abb. 3: Einflussfaktoren Verbrauchsmenge, Humanmetabolismus und Kläranlageneliminierung auf die PEC-BRB 
Tabelle 6: Ableitung der PNEC aus ökotoxikologischen Wirkungswerten ${ }^{e}$

\begin{tabular}{|c|c|c|c|c|c|c|}
\hline Wirkstoff & $\begin{array}{c}\text { Molare } \\
\text { Masse } \\
{[\mathrm{g} / \mathrm{mol}]}\end{array}$ & $\begin{array}{l}\text { Charakterisierung des niedrigsten bekannten } \\
\text { Wirkungswertes }\end{array}$ & $\begin{array}{c}\text { niedrigster } \\
\text { Wirkungswert } \\
{[\mu \mathrm{g} / \mathrm{l}]}\end{array}$ & Quelle & $\begin{array}{l}\text { Sicher- } \\
\text { heits- } \\
\text { faktor }\end{array}$ & $\begin{array}{l}\text { PNEC } \\
{[\mu \mathrm{g} / \mathrm{I}]}\end{array}$ \\
\hline Acetylsalicylsäure & 180 & Bakterientoxizität, $\mathrm{EC}_{0}$, Pseudomonas putida & 8000 & [17] & 100 & 80 \\
\hline Benzalkoniumchlorid & $280-420$ & Algentoxizität, $\mathrm{EC}_{50}$, Scenedesmus subspicatus & 24 & [15] & 200 & 0,12 \\
\hline Carbamazepin & 236 & Daphnientoxizität, NOEC C.dubia, $7 \mathrm{~d}$ & 25 & [56] & $100^{f}$ & 0,25 \\
\hline Ciprofloxacin-HCl & 368 & Bakterientoxizität, $\mathrm{EC}_{10}$, Pseudomonas putida & 1,8 & [18] & 100 & 0,018 \\
\hline Clofibrinsäure & 215 & Daphnientoxizität, NOEC Reproduktion, & 10 & [1] & $100^{f}$ & 0,1 \\
\hline Diclofenac-Na & 318 & Daphnientoxizität, NOEC, 7 d, C. dubia & 1000 & [56] & $100^{1}$ & 10 \\
\hline Dodecylbispropylentriamin & 300 & Daphnientoxizität, $\mathrm{EC}_{50}, 24 \mathrm{~h}$, Daphnia magna & $<1000$ & [19] & 200 & $<5$ \\
\hline Formaldehyd & 30 & Algentoxizität, Scenedesmus & $300-500$ & [15] & 200 & 1,5 \\
\hline Glutaral & 100 & Algentoxizität, $\mathrm{EC}_{50}, 72 \mathrm{~h}$ & 610 & [20] & 200 & 3,05 \\
\hline Glyoxal & 58 & Bakterientoxizität, $\mathrm{EC}_{50}$ Wachstum, Pseudomonas putida & 133700 & [15] & 200 & 668 \\
\hline Ibuprofen & 206 & Daphnientoxizität, NOEC, Daphnia magna & 3000 & [21] & 100 & 30 \\
\hline lodixanol & 1550 & Daphnientoxizität, $\mathrm{EC}_{50}$ & $>2500000$ & [22] & 200 & $>12500$ \\
\hline Paracetamol & 151 & Daphnientoxizität, $\mathrm{EC}_{50}, 48 \mathrm{~h}$, Daphnia magna & 9200 & [23] & 200 & 46 \\
\hline Acarbose & 646 & Daphnientoxizität, $\mathrm{EC}_{0}$, Daphnia magna & $>1000000$ & [24] & 500 & $>2000$ \\
\hline Cyclophosphamid & 261 & Fischtoxizität, NOEC 96 h, Salmo gairdneri & $>984000$ & [23] & 500 & $>1970$ \\
\hline Ethinylestradiol & 296 & $\begin{array}{l}\text { Fischtoxizität, NOEC full life-cycle-test, } \\
\text { Geschlechtsentwicklung, Wachstum, histologische } \\
\text { Organveränderungen, Pimephales promelas }\end{array}$ & 0,001 & {$[52]$} & $500^{\prime}$ & 0,000002 \\
\hline Glucoprotamin & & Daphnientoxizität, $\mathrm{EC}_{50}$ & 500 & [15] & 1000 & 0,5 \\
\hline Ifosfamid & 261 & Daphnientoxizität, NOEC 48 h, Daphnia magna & 100000 & [28] & 500 & 200 \\
\hline Lidocain $\mathrm{HCl}$ & 271 & Fischtoxizität, LC $_{50}, 96$ h, Zebrafisch & 106000 & [29] & 1000 & 106 \\
\hline Mezlocillin-Na-Monohydrat & 562 & Daphnientoxizität, Daphnia magna Strauss & 20000 & [30] & 1000 & 20 \\
\hline Pentaerythrithyltetranitrat & 316 & Bakterientoxizität, $\mathrm{EC}_{50}, 30 \mathrm{~min}$, Vibrio fischeri & 14500 & [31] & 1000 & 14,5 \\
\hline Prilocain- $\mathrm{HCl}$ & 257 & Daphnientoxizität, $\mathrm{EC}_{50}, 48$ h, Daphnia magna & 61000 & [32] & 1000 & 61 \\
\hline Verapamil-HCl & 491 & Fischtoxizität, LC $_{50}, 96$ h, Goldorfe & $4600-10000$ & [33] & 1000 & $4,6-10$ \\
\hline 5-Fluorouracil & 130 & Akute Daphnientoxizität, $\mathrm{EC}_{10}$, Daphnia magna & 1300 & [57] & 2500 & 0,52 \\
\hline Furosemid & 331 & Fischtoxizität, LC $_{50}, 96$ h, Goldorfe & $>500000$ & [35] & 5000 & $>100$ \\
\hline Laurylpropylendiamin & 243 & Fischtoxizität, LC $_{50}, 96$ h, Zebrabärbling & 100 & [36] & 5000 & 0,02 \\
\hline Metamizol-Na & 334 & Fischtoxizität, LC $_{50}, 96$ h, Goldorfe & $>500000$ & [37] & 5000 & $>100$ \\
\hline Metformin- $\mathrm{HCl}$ & 166 & Daphnientoxizität, $\mathrm{EC}_{50}, 48$ h, Daphnia m. & 60000 & [38] & 5000 & 12 \\
\hline Naproxen & 230 & Daphnientoxizität, $\mathrm{EC}_{50}, 24$ h, Daphnia m. & 140000 & [27] & 5000 & 28 \\
\hline Norethisteron & 298 & Daphnientoxizität, $\mathrm{EC}_{50}, 48 \mathrm{~h}$, Daphnia magna & $>4600$ & [39] & 5000 & $>0,92$ \\
\hline Pentoxifyllin & 278 & Fischtoxizität, LC ${ }_{50}, 96$ h, Zebrabärbling & 100000 & [40] & 5000 & 20 \\
\hline Polyvidon-lod & 2252 & Fischtoxizität, NOEC, Leuciscus idus & 4600 & [41] & 2500 & 1,84 \\
\hline Propranolol-HCl & 296 & Daphnientoxizität, $\mathrm{EC}_{50}$, Daphnia magna & 2700 & [27] & 5000 & 0,54 \\
\hline Propyphenazon & 230 & Fischtoxizität, LC $_{50}, 96 \mathrm{~h}$, Zebrabärbling & 220000 & [42] & 5000 & 44 \\
\hline Clarithromycin & 748 & $\begin{array}{l}\text { Bakterientoxizität, } \mathrm{EC}_{50}, \text { Wachstum, } \\
\text { Enterecoccus faecalis }\end{array}$ & 151 & [43] & 25000 & 0,006 \\
\hline $\begin{array}{l}\text { Cocospropylendiamin- } \\
\text { guaniacetat }\end{array}$ & & Fischtoxizität, LC Lo, 96 h, Zebrabärbling & $<1000$ & [44] & 25000 & $<0,04$ \\
\hline Naftidrofurylhydrogenoxalat & 474 & Daphnientoxizität, $\mathrm{EC}_{50}, 48 \mathrm{~h}$ & 20000 & [45] & 25000 & 0,8 \\
\hline Phenazon & 188 & Fischtoxizität, $\mathrm{LC}_{50}, 96 \mathrm{~h}$ & $>500000$ & [46] & 25000 & $>20$ \\
\hline Roxithromycin & 837 & Fischtoxizität, $\mathrm{LC}_{50}, 96 \mathrm{~h}$ & $>100000$ & [47] & 25000 & $>4$ \\
\hline Theophyllin & 180 & Daphnientoxizität, $\mathrm{EC}_{50}, 24 \mathrm{~h}$ & 155000 & [27] & 25000 & 6,2 \\
\hline
\end{tabular}

${ }^{\theta}$ : Die innerhalb der Tabelle erfolgte Grauabstufung der Spaltenbereiche weist auf den unterschiedlichen ökotoxikologischen Datenfundus bezüglich der Trophiestufenzahl im aquatischen Ökosystem hin. Der Übergang von weiß nach dunkelgrau entspricht der Verfügbarkeit von ökotoxikologischen Wirkungsdaten zwischen vier und einer Trophiestufe(n) und spiegelt sich in der Wahl der Sicherheitsfaktoren (siehe Tab. 4) wider.

: Da für diese Wirkstoffe Ergebnisse aus chronischen Tests vorliegen, sind die Sicherheitsfaktoren möglicherweise zu hoch. Dennoch liegen uns z.B. keine Ergebnisse zur Bakterientoxizität von Ethinylestradiol vor, so dass für die Bewertung eine trophische Stufe fehlt. Wir haben uns deshalb entschlossen, den dargestellten Bewertungsformalismus auch für diese Wirkstoffe beizubehalten. Die Gesamtaussage (Verhältnis PEC-BRB : PNEC) würde für Ethinylestradiol und Diclofenac-Na auch bei kleineren Sicherheitsfaktoren erhalten bleiben, während sich für Clofibrinsäure und Carbamazepin das Verhältnis PEC-BRB:PNEC auf einen Wert $<1$ verringern würde . 
aus den üblichen Tests zur akuten Fisch-, Daphnien- und Algentoxizität resultierenden Daten die tatsächliche Ökotoxizität unter Umständen stark unterschätzt wird.

\subsection{Bewertung der Umweltrelevanz}

Für etwa 2/3 der ursprünglich betrachteten Wirkstoffe konnte eine Bewertung nach dem PEC : PNEC-Konzept vorgenommen werden, da, allerdings in unterschiedlichem Umfang, ökotoxikologische Wirkungsdaten zur Verfügung standen. Die entsprechenden PEC-BRB : PNEC-Verhältnisse sind in Abb. 4 (für Stoffe mit Wirkungsdaten für vier und drei Trophiestufen) sowie in Abb. 5 (für Stoffe mit Wirkungsdaten für zwei bzw. eine Trophiestufe(n)) dargestellt.

Somit lässt sich nach dem Kriterium ökotoxikologischer Wirkungen, aber auch unter Betrachtung sonstiger Umwelteigenschaften für die Antibiotika Ciprofloxacin- $\mathrm{HCl}$ und Clarithromycin, das Antiepileptikum Carbamazepin, die Desinfektionsmittelwirkstoffe Benzalkoniumchlorid, Cocospropyl- endiaminguaniacetat, Glucoprotamin, Laurylpropylendiamin und Polyvidon-Iod, das Sexualhormon Ethinylestradiol, das Antidiabetikum Metformin- $\mathrm{HCl}$ sowie für den Lipidsenkermetaboliten Clofibrinsäure ein Umweltgefährdungspotential für Brandenburger Oberflächengewässer ableiten, da deren PEC-BRB : PNEC-Verhältnisse um 1 oder darüber liegen.

Nachfolgend sollen exemplarisch die Resultate verschiedener Risikoabschätzungen (PEC : PNEC-Verhältnisse) für die Wirkstoffe Acetylsalicylsäure, Clofibrat/Clofibrinsäure und Ethinylestradiol verglichen werden.

Tabelle 7 verdeutlicht, dass verschiedene Risikobewertungen für ein und denselben Arzneimittelwirkstoff zu übereinstimmenden, aber auch, wie insbesondere das Beispiel Ethinylestradiol zeigt, zu durchaus unterschiedlichen Resultaten führen können. Eine Vergleichbarkeit der Resultate aus verschiedenen Risikoabschätzungen ist nur gegeben, wenn die Methodik und die Datenbasis bei der Definition der PEC (Verbrauchsmengen, Humanmetabolismus, Kläranlagenverhalten, Verdün-

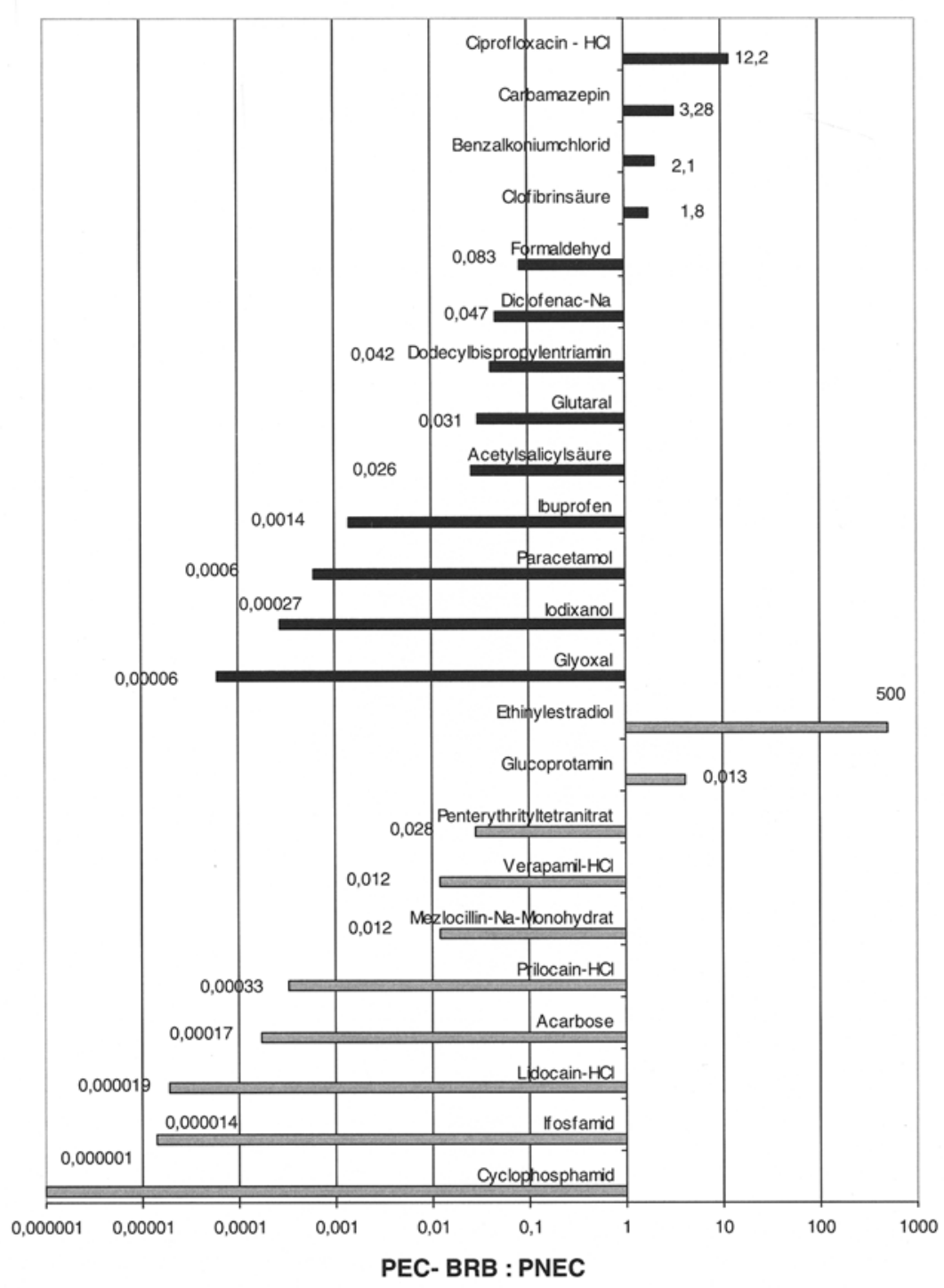

Abb. 4: PEC-BRB : PNEC-Verhältnisse ( Wirkungsdaten für vier und drei Trophiestufen) 


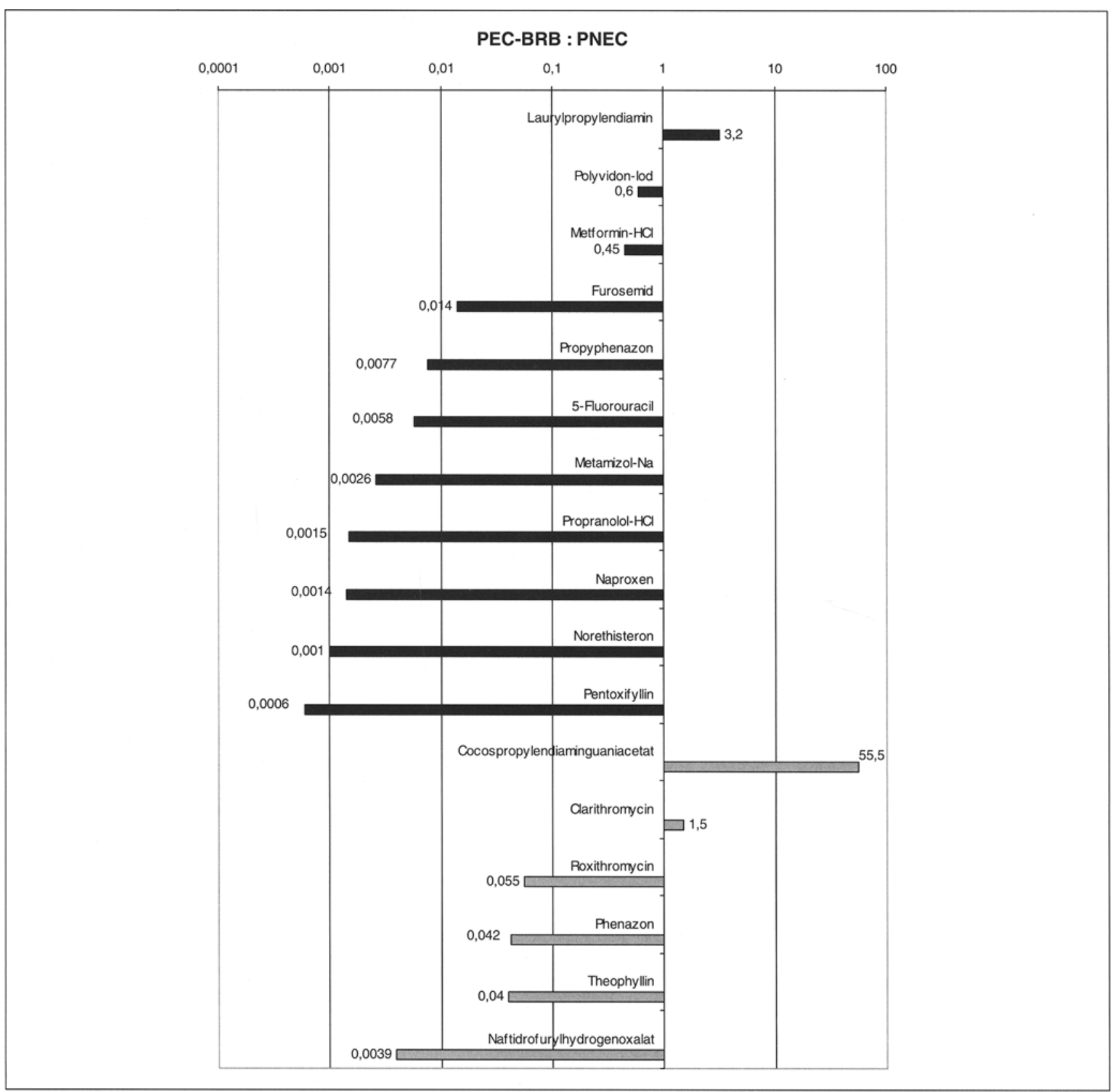

Abb. 5: PEC : PNEC-Verhältnisse (Wirkungsdaten für zwei und eine Trophiestufe(n))

Tabelle 7: Vergleich PEC : PNEC nach verschiedenen Risikobewertungen

\begin{tabular}{l|c|c|c}
\hline Wirkstoff & $\begin{array}{c}\text { Landesumweltamt } \\
\text { Brandenburg }\end{array}$ & Römbke et al. [54] & Webb [53] \\
\hline Acetylsalicylsäure & 0,013 & 0,002 & $<0,01$ \\
\hline $\begin{array}{l}\text { Clofibrat / } \\
\text { Clofibrinsäure }\end{array}$ & $\begin{array}{c}1,8 \\
\text { (chronische Wirkdaten) }\end{array}$ & $\begin{array}{c}8,75 \\
\text { (chronische Wirkdaten) }\end{array}$ & $\begin{array}{c}0,02 \text { (akute Wirkdaten) } \\
3,6(90-P)-8,75 \text { (max. Messwert) } \\
\text { (chronische Wirkdaten) }\end{array}$ \\
\hline Ethinylestradiol & $\begin{array}{c}500 \\
\text { (endokrine Effekte berücksichtigt, } \\
\text { PEC worst case, } \\
\text { hoher Sicherheitsfaktor, } \\
\text { siehe auch Fußnote', Tabelle 6) }\end{array}$ & $\begin{array}{c}1->2 \text { bzw. <1 } \\
\text { (endokrine Effekte nicht } \\
\text { berücksichtigt, sehr hohe PEC) }\end{array}$ & $\begin{array}{c}0,62 \\
\text { (geringe (1:2) bzw. größere (1:10) Verdünnung, } \\
\text { endokrine Effekte berücksichtigt, relativ geringe PEC) }\end{array}$ \\
\hline
\end{tabular}


Tabelle 8: Prognose der Umweltrelevanz für Wirkstoffe ohne verfügbare ökotoxikologische Wirkungsdaten (Kriterien siehe 1.4)

\begin{tabular}{|c|c|c|c|}
\hline $\begin{array}{l}\text { Gruppe 1: } \\
\text { Umweltrelevanz wahrscheinlich }\end{array}$ & $\begin{array}{l}\text { nach } 1.4 \\
\text { erfüllte } \\
\text { Kriterien }\end{array}$ & $\begin{array}{l}\text { Gruppe 2: } \\
\text { Umweltrelevanz wenig oder nicht } \\
\text { wahrscheinlich }\end{array}$ & $\begin{array}{l}\text { Gruppe 3: } \\
\text { Umweltrelevanz mangels Daten } \\
\text { derzeit nicht prognostizierbar }\end{array}$ \\
\hline 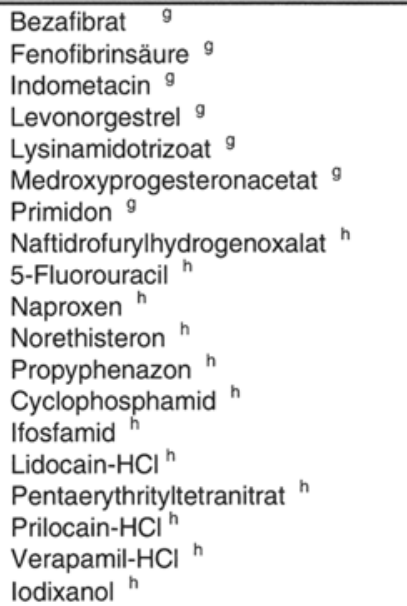 & $\begin{array}{l}\mathrm{a}, \mathrm{e} \\
\mathrm{a}, \mathrm{e} \\
\mathrm{a}, \mathrm{e} \\
\mathrm{a}, \mathrm{e} \\
\mathrm{c}, \mathrm{d} \\
\mathrm{a}, \mathrm{e} \\
\mathrm{c}, \mathrm{e} \\
\mathrm{a}, \mathrm{c} \\
\mathrm{c}, \mathrm{e} \\
\mathrm{a}, \mathrm{c}, \mathrm{e} \\
\mathrm{a}, \mathrm{c}, \mathrm{e} \\
\mathrm{c}, \mathrm{e} \\
\mathrm{c}, \mathrm{d}, \mathrm{e} \\
\mathrm{c}, \mathrm{e} \\
\mathrm{c}, \mathrm{e} \\
\mathrm{a}, \mathrm{c} \\
\mathrm{c}, \mathrm{e} \\
\mathrm{a}, \mathrm{c} \\
\mathrm{c}, \mathrm{d}\end{array}$ & $\begin{array}{l}\text { Acetylcystein } \\
\text { Allopurinol } \\
\text { Isosorbiddinitrat } \\
\text { Metoprololtartrat }\end{array}$ & $\begin{array}{l}\text { Ampicillin } \\
\text { Captopril } \\
\text { Dimeticon } \\
\text { Phenoxypropanol-Isomere } \\
\text { Piperacillin-Na } \\
\text { Prednisolon } \\
\text { Sulbactam-Na } \\
\text { Sultamicillin } \\
\text { Torasemid-Na }\end{array}$ \\
\hline
\end{tabular}

nungsfaktor) sowie bei der Ableitung der PNEC (akute bzw. chronische Wirkdaten, Sicherheitsfaktoren) vergleichbar sind. Dies ist in der o.g. Gegenüberstellung nur bedingt der Fall.

Für ca. 1/3 der in dieser Arbeit betrachteten 60 Wirkstoffe liegen derzeit keine ökotoxikologischen Wirkkonzentrationen vor. Auch wenn dadurch eine Bewertung der ökotoxikologischen Relevanz im aquatischen Ökosystem für diese Stoffe nicht möglich ist, soll nachfolgend entsprechend den in 1.4 benannten Kriterien eine grobe Einteilung in drei Gruppen versucht werden. Die Zuordnung der Wirkstoffe in diese Gruppen ist Tabelle 8 zu entnehmen.

1. Gruppe: Wirkstoffe, für die eine Umweltrelevanz als
wahrscheinlich angesehen werden kann

Für die in diese Gruppe eingeteilten Wirkstoffe sind mindestens zwei der in 1.4 genannten Umweltrelevanzkriterien erfüllt. Dabei enthält diese Gruppe sowohl Stoffe, für die keine ökotoxikologischen Wirkungswerte verfügbar waren (in Tab. 8 mit g gekennzeichnet) als auch solche Stoffe, für die nach dem PEC : PNEC-Konzept zwar rein wirkungsseitig keine Umweltrelevanz ermittelt werden konnte, die jedoch ebenfalls mindestens zwei Kriterien nach 1.4 erfüllen (in Tab. 8 mit h ${ }^{\text {gekennzeichnet). }}$

2. Gruppe: Wirkstoffe, für die es derzeit wenig oder keine Hinweise für eine ökotoxikologische Relevanz gibt

3. Gruppe: Wirkstoffe, für die mangels verfügbarer Daten zum Umweltverhalten eine Prognose der Umweltrelevanz derzeit nicht möglich ist

Bisher konnte für keinen der bewerteten Stoffe eine Halbwertszeit von mehr als 2 Monaten im Oberflächenwasser (Kriterium b) ermittelt werden, wobei jedoch für viele Arzneimittelwirkstoffe keine entsprechenden Halbwertszeiten verfügbar waren.

Inwieweit Daten aus QSAR-Rechnungen bei der Abschätzung des Umweltverhaltens verwendet werden können, soll nachfolgend kurz am Beispiel der berechneten minimalen Fischtoxizitäten (durch Vergleich mit experimentell bestimmten Werten, siehe Tabelle 9) gezeigt werden.

Tabelle 9: Vergleich von experimentell ermittelten Fischtoxizitäten mit den nach der Könemann-Gleichung berechneten minimalen Fischtoxizitäten (alle Angaben als $\mathrm{LC}_{50}$ in $\mathrm{mg} / \mathrm{l}$ )

\begin{tabular}{|c|c|c|c|}
\hline Wirkstoff & $\log K_{\text {ow }}$ berechnet & LC $_{50}$ experimentell ermittelt & $\begin{array}{c}\text { LC }_{50} \text { nach der } \\
\text { Könemann-Gleichung } \\
\text { berechnet }\end{array}$ \\
\hline Propyphenazon & 1,7 & $\begin{array}{c}\mathbf{2 2 0 - 5 5 0} \\
\text { (Test nach OECD } 203 \text { am Zebrabärbling) [42] }\end{array}$ & 564 \\
\hline Clofibrinsäure & 3,1 & $\begin{array}{c}\mathbf{8 6} \\
\text { (Sterblichkeit Fischembryonen, Test nach Schulte und Nagel) [1] }\end{array}$ & 53,1 \\
\hline Benzalkoniumchlorid & 3,4 & $\begin{array}{c}\mathbf{5 , 2} \\
(\text { Leuciscus idus })[15]\end{array}$ & 27,6 \\
\hline Laurylpropylendiamin & 4,8 & $\begin{array}{c}\mathbf{0 , 1 - 0 , 3 5} \\
\text { (Zebrabärbling, 96h) [36] }\end{array}$ & 1,2 \\
\hline Ibuprofen & 3,7 & $\begin{array}{c}173 \\
\text { (Lepomis machrochirus, } 96 \mathrm{~h})[21]\end{array}$ & 8,5 \\
\hline Naproxen & 3,0 & $\begin{array}{c}\mathbf{5 6 0} \\
(\text { Lepomis machrochirus, } 96 \text { h) [27] }\end{array}$ & 45 \\
\hline Paracetamol & 0,4 & $\begin{array}{c}\mathbf{3 7 8} \\
\text { (Sterblichkeit Fischembryonen, Test nach Schulte und Nagel) [1] }\end{array}$ & 5208 \\
\hline Pentoxifyllin & 0,3 & $\begin{array}{c}\mathbf{1 0 0 - 2 2 0} \\
(\text { Zebrabärbling, Test nach OECD 203, } 96 \text { h) [40] }\end{array}$ & 10000 \\
\hline
\end{tabular}


Ein Vergleich der mit der Könemann-Gleichung ermittelten Mindestfischtoxizitäten mit den der Literatur zu entnehmenden, experimentell ermittelten $\mathrm{LC}_{50}$-Werten liefert ein insgesamt heterogenes Bild: Für einige Wirkstoffe mit mittlerem bis hohem $\log K_{\text {ow }}$, wie z.B. für Clofibrinsäure, Propyphenazon oder für Benzalkoniumchlorid und Laurylpropylendiamin erhält man eine relativ gute Übereinstimmung (Differenz $<$ Faktor 5). Für eine weitere Substanzgruppe mit hohem $\log K_{\text {ow }}$ ( wie z.B. Naproxen oder Ibuprofen) ist die mittels QSAR berechnete Mindestfischtoxizität deutlich höher als die bisher experimentell ermittelte. Mögliche Gründe hierfür könnten in unterschiedlichen Prüfbedingungen bzw. in Variationen der Sensitivität sowie der metabolischen Kapazität der Testorganismen zu finden sein. Für eine dritte Wirkstoffgruppe (hier beispielhaft durch Paracetamol und Pentoxifyllin vertreten) sind die experimentell ermittelten $\mathrm{LC}_{50}$-Werte deutlich geringer als die berechneten. Dieser Sachverhalt weist auf das Vorhandensein spezifischer Toxizitätsmechanismen hin, für welche die Könemann-Gleichung (abgesehen von Spezies-Unterschieden) erwartungsgemäß eine Unterschätzung der aquatischen Toxizität liefert. Zugleich verfügen diese Wirkstoffe über eine geringere Lipophilie, was an den $\log K_{\text {ow }}$-Werten erkennbar ist.

\subsection{Analysenempfehlungen}

Gemäß der in 1.5 vorgenommenen Einteilung ergeben sich hinsichtlich der Stoffanalytik in Brandenburger Oberflächengewässern folgende Prioritäten:

Priorität A mit der Empfehlung zur Routineanalytik für die Antibiotika Ciprofloxacin-HCl und Clarithromycin, das Antiepileptikum Carbamazepin, die Desinfektionsmittelwirkstoffe Benzalkoniumchlorid, Cocospropylendiaminguaniacetat, Glucoprotamin, Laurylpropylendiamin und Polyvidon-Iod, das Sexualhormon Ethinylestradiol, das Antidiabetikum Metformin- $\mathrm{HCl}$ sowie für den Lipidsenkermetaboliten Clofibrinsäure. Priorität B mit der Empfehlung der Analytik an ausgewählten Messstellen (z.B. hoher Abwasseranteil) für die in Tabelle 8 in Gruppe 1 erwähnten Stoffe.

Priorität $\mathrm{C}$ mit der Analysenempfehlung für erweiterte Messprogramme an wenigen Messstellen in größerem Zeitraster für die in Tabelle 8 in Gruppe 3 erwähnten Stoffe.

Priorität 0 für alle betrachteten Stoffe außerhalb der Prioritäten $\mathrm{A}$ bis $\mathrm{C}$.

\section{Ausblick}

Den Autoren ist durchaus bewusst, dass nicht zuletzt wegen des im Vergleich zu Industriechemikalien oder Pflanzenschutzmitteln bei Arzneimittelwirkstoffen spärlichen Vorhandenseins valider Wirkdaten eine umfassende Abschätzung des Umweltgefährdungspotentials in vergleichbarer Qualität derzeit nicht möglich ist. So ist zu bedenken, dass die aus den ökotoxikologischen Standardtestverfahren (meist Akuttests) gewonnenen Daten chronische Effekte nicht berücksichtigen und somit das ökosystemare Umweltgefährdungspotential möglicherweise unterschätzt wird [1]. Ferner muss das Problem der Kombinationswirkungen verschiedener, in der aquatischen Umwelt nebeneinander existierender Wirkstoffe berücksichtigt werden [57].

Trotz der genannten vielfältigen Einschränkungen erscheint es nach unserer Einschätzung nicht nur gerechtfertigt son- dern auch notwendig, eine erste und orientierende Bewertung von möglichen arzneimittelbedingten Risiken für aquatische Ökosysteme vorzunehmen, auch wenn uns bewusst ist, das diese Bewertung zunächst tendenziellen Charakter hat und im Zuge zu erwartender und zu fordernder neuer wissenschaftlicher Erkenntnisse fort- und möglicherweise auch umgeschrieben werden wird.

Eine Möglichkeit dazu bietet der nach Abfassung dieser Arbeit veröffentlichte und seit Juli 2003 in der Kommentierungsphase befindliche überarbeitete Entwurf des EMEA-Leitfadens [55]. In diesem wird die Bewertung der Exposition in zwei Phasen, zunächst als worst-case-Szenario und bei Überschreitung des Triggerwertes von $0,01 \mu \mathrm{g} / \mathrm{l}$ nach einem verfeinerten Verfahren unter Berücksichtigung einer genaueren Marktanalyse sowie des Umweltverhaltens und des Metabolismus des Wirkstoffes durchgeführt. Ein Vergleich der nach den PECFormeln des alten und des neuen EMEA-Leitfadens berechneten PEC worst case zeigt nicht zwangsläufig unterschiedliche Ergebnisse. Wir haben stichprobenartig die PEC für Metoprolol, Paracetamol und Ibuprofen nach der alten und der neuen Formel auf der Basis der in den Fachinformationen genannten maximalen Tagesdosen bzw. der Verkaufszahlen für 2001 in Deutschland (Quelle: IMS/UBA [58]) verglichen. Resultat alte PEC vs. neue PEC: Metoprolol: 2,0 $\mu \mathrm{g} / 1 \mathrm{vs.} 2,25 \mu \mathrm{g} / 1$; Paracetamol: 13,7 $\mu \mathrm{g} / \mathrm{l}$ vs. 17,5 $\mu \mathrm{g} /$; Ibuprofen: 7,63 $\mu \mathrm{g} / \mathrm{l}$ vs. $12 \mu \mathrm{g} /$. Bezüglich der Wirkungsanalyse werden zur Ableitung der PNEC in Abhängigkeit vom Vorhandensein chronischer Testdaten Sicherheitsfaktoren zwischen 10 und 1000 vorgeschlagen. Eine PNEC für Mikroorganismen wird dabei separat abgeleitet. Ferner gibt es auch Vorschläge für ein Risk-Assessment für den terrestrischen Bereich. Eine interessante Aufgabe könnte u.E. nun darin bestehen, die in dieser Arbeit diskutierten Wirkstoffe bei Vorhandensein entsprechender Effektdaten nach der Methodik des neuen EMEA-Leitfadens zu bewerten und die mit beiden Methoden erhaltenen Ergebnisse zu vergleichen.

Danksagung. Besonders gedankt sei allen pharmazeutischen Unternehmen, deren namentliche Aufzählung an dieser Stelle den Rahmen sprengen würde, für ihr Interesse an dieser Problematik und für die engagierte Unterstützung bei der Beschaffung von Stoffdaten in Form von Sicherheitsdatenblättern, Fachinformationen und anderer Literatur, welche zu großen Teilen die Datenbasis zur Beurteilung der Ökotoxizität bildeten. Für die Mitarbeit bei der Gewinnung der QSAR-Daten danken wir Herrn Ralf-Uwe Ebert und Herrn Dr. Ralph Kühne.

\section{Literatur}

[1] Ternes Th, Hirsch R, Stumpf M, Eggert T, Schuppert B, Haberer K (1999): Nachweis und Screening von Arzneimittelrückständen, Diagnostika und Antiseptika in der aquatischen Umwelt. Abschlussbericht des ESWE-Institutes für Wasserforschung und Wassertechnologie $\mathrm{GmbH}$ zum Forschungsvorhaben 02WU9567/3 des BMBF $234 \mathrm{~S}$.

[2] Heberer Th, Stan HJ (1997): Determination of clofibric acid and $\mathrm{N}$-(phenylsulfonyl)-sarcosine in sewage, river and drinking water. Inter J Environ Anal Chem 67, 113-124

[3] Heberer Th, Schmidt-Bäumler K, Stan HJ (1998): Occurrence and Distribution of Organic Contaminants in the Aquatic System in Berlin. Part I. Drug Residues and other Polar Contaminants in Berlin Surface and Groundwater. Acta hydrochim hydrobiol 26 (5) 272-278

[4] Kratz W, Abbas B, Linke I (2000): Arzneimittelwirkstoffe in der Umwelt. UWSF - Z Umweltchem Ökotox 12 (6) 343-349

[5] Abbas B, Kratz W (2000): Humanarzneimittel in der Umwelt. Erhebung von Humanarzneimittelmengen im Land Brandenburg 1999. Studien und Tagungsberichte Band 25. Hrsg.: Landesumweltamt Brandenburg 
[6] Abbas B, Linke I, Kratz W (2001): Erhebung des Verbrauchs von Arzneimitteln im Land Brandenburg. UWSF - Z Umweltchem Ökotox 13 (4) 197-203

[7] www.rote-liste.de

[8] BLAC (2001): Hrsg.: Bund-Länderausschuss für Chemikaliensicherheit. Untersuchungsprogramm Arzneimittel in der Umwelt. unveröffentlicht. $170 \mathrm{~S}$.

[9] Fuhrmann B (1999): Vergleichende Untersuchung von Oberflächenwässern in Athen und Berlin auf Arzneimittelrückstände aus kommunalen Kläranlagen. Diplomarbeit. Institut für Lebensmittelchemie der TU Berlin, $77 \mathrm{~S}$.

[10] EMEA (2001): The European Agency for the Evaluation of Medicinical Products, Discussion Paper on Environmental Risk Assessment of non-genetically modified Organism (non-GMO) containing Medicinical Products for Human Use. London. 13 pp

[11] Breitkopf C, Kühne R, Schüürmann G (2000): Dependence of Multimedia Level-III Partitioning and Residence Times of Compounds on Physicochemical Properties and System Parameters of Water-Rich and Water-Poor Environments. Environ Toxicol Chem $19,1430-1440$

[12] Schüürmann G (2001): Stoffeigenschaften zu 57 ArzneimittelInhaltsstoffen. Umweltforschungszentrum Leipzig-Halle $\mathrm{GmbH}$, Sektion Chemische Ökotoxikologie. Schriftliche Mitteilungen vom 18.09.2001 und 12.12.2001, unveröffentlicht

[13] Schüürmann G, Marsmann M (1991): QSAR-Modelle - Interpretation und Prognose der Biokonzentration und aquatischen Toxizität. UWSF - Z Umweltchem Ökotox 3 (1) 42-47

[14] http://www.chem.unep.ch/pops/newlayout/negotriatons.htm

[15] Gartiser S, Stiene G, Hartmann A, Zipperle J (1999): Umweltverträgliche Desinfektionsmittel in Krankenhausabwässern. Hydrotox GmbH Freiburg im Auftrag des Umweltbundesamtes. FKZ 29727526, 106 S.

[16] Möhle E, Kempter C, Kern A, Metzger JW (1999): Untersuchungen zum Abbau von Pharmaka in kommunalen Kläranlagen mit HPLC-Electrospray-Massenspektrometric. Acta hydrochim hydrobiol 27 (6) 430-436

[17] Merck KgaA Darmstadt (2000): Sicherheitsdatenblatt Acetylsalicylsäure

[18] Bayer AG (2001): Sicherheitsdatenblatt Ciprofloxacin-Hydrochlorid

[19] Lonza AG Basel (1997): Sicherheitsdatenblatt Lonzabac 12.100

[20] Dr. Theodor Schuchardt \& Co. (1999): Sicherheitsdatenblatt Glutardialdehyd (25\%-ige Lösung zur Synthese)

[21] Halling-Sørensen B, Nielsen Nors S, Lansky PF, Ingerselv F, Holten Lützhøft HC, Jørgensen SE (1997): Occurrence, Fate and Effects of Pharmaceutical Substances in the Environment - A Review. Hrsg: The Royal Danish School of Pharmacy 357-391

[22] Nycomed Amersham Buchler GmbH \& Co. KG (2001): Umweltdatenblatt Visipaque ${ }^{\circledR}$ Iodixanol

[23] Stuer-Lauridsen F, Birkved M, Hansen LP, Holten Lützhøft HC, Halling-Sørensen B (2000): Environmental risk assessment of human pharmaceuticals in Denmark after normal therapeutic use. Chemosphere 40, 783-793

[24] Bayer AG (1999): Sicherheitsdatenblatt Acarbose 050192/09, 1999

[25] ASTA Medica AG (2001): Sicherheitsdatenblatt Cyclophosphamid

[26] BIOCHEMIE Ges.m.b.H., Kundl (1999): Sicherheitsdatenblatt Diclofenac-Natrium

[27] Kümmerer K (Hrsg.) (2001): Pharmaceuticals in the Environment - Fate, Effects and Risks. Springer Verlag Berlin, $265 \mathrm{pp}$

[28] Schecker J, Al-Ahmad A, Bauer M, Zellmann H, Kümmerer $\mathrm{K}$ (1998): Elimination des Zytostatikums Ifosfamid während der simulierten Zersetzung von Hausmüll im Labormaßstab. UWSF - Z Umweltchem Ökotox 10 (6) 339-344

[29] Astra Zeneca GmbH (2000): Sicherheitsdatenblatt Lidocaine Hydrochloride

[30] Bayer AG (2001): Sicherheitsdatenblatt Baypen p.i./Mezlocillin

[31] Rippen G (Hrsg.) (1990): Handbuch Umweltchemikalien. Stoffdaten-Prüfverfahren-Vorschriften. ecomed verlag Landsberg - Loseblatt-Ausgabe, 3. Auflage 1990, Bd. 4-8

[32] Astra Zeneca GmbH (2000): Sicherheitsdatenblatt Prilocaine Hydrocloride

[33] Knoll GmbH Ludwigshafen (1997): Sicherheitsdatenblatt Verapamil-HCl

[34] Hanisch B, Abbas B, Kratz W (2002): Ökotoxikologische Bewertung von Humanarzneimitteln in aquatischen Ökosystemen. Studien und Tagungsberichte Band 39. Hrsg.: Landesumweltamt Brandenburg
[35] Hoechst Marion Roussel Deutschland GmbH (1999): Sicherheitsdatenblatt Furosemid

[36] Clariant GmbH Frankfurt / M. (2000): Sicherheitsdatenblatt Genamin LAP $100 \mathrm{D}$

[37] Höchst Marion Roussel (1999): Sicherheitsdatenblatt Metamizol$\mathrm{Na}$ Monohydrat

[38] Merck Lipha (1997): Material Safety Data Sheet Metforminhydrochlorid

[39] Schering AG Berlin (2000): Sicherheitsdatenblatt Norethisteron

[40] Aventis Pharma (2000): Sicherheitsdatenblatt Pentoxyfyllin

[41] BASF AG (2001): Sicherheitsdatenblatt PVP-Iod

[42] Hoechst Marion Roussel (1999): Sicherheitsdatenblatt Propyphenazon. Version 1.1, 1999

[43] Alexy R, Kümpel T, Kümmerer K (2001): Effekte von Antibiotika auf das Bakterienwachstum in der Umwelt. (SETAC-Tagung Berlin 10.09.2001), Abstractband

[44] Dr. Schuhmacher GmbH (1995): Sicherheitsdatenblatt Cocospropylen-1,5-bis-guanidiniumacetat

[45] Merck Lipha s.a. France (1997): Safety Data Sheet, Naftidrofuryl Oxalate, Edition Nr. 2

[46] Kraemer \& Martin Pharma Handels GmbH (1998): Sicherheitsdatenblatt Phenazon

[47] Hoechst Marion Roussel (1999): Roxithromycin. Safety Data Sheet

[48] Allner B, Bergholz A, Möller M, Stahlschmidt-Allner P, Wegener G (1998): Wirkungen umweltrelevanter synthetischer Ostrogene auf Fische. Fachtagung 'Arzneimittel in Gewässern - Risiko für Mensch, Tier und Umwelt?' 04. Juni 1998 Wiesbaden. Hessische Landesanstalt für Umwelt

[49] Braunbeck Th, Islinger M, Koerr S, Pawlowski S, Bieberstein U, Hollert H, Siebert I, Uhde Th, Wetterauer B (2001): Endocrine disrupters in fish and fish cells - in vitro versus in vivo testing strategies. Universität Heidelberg. Second Status Seminar endocrine disrupters. 02.-04.04.2001 Berlin. Proceedings 89-92

[50] Schäfers Ch, Wenzel A, Schmitz A, Böhmer W (2001): Effects of xenoestrogens on the life cycle of fish. Fraunhofer-Institut für Umweltchemie und Ökotoxikologie IUCT. Second Status Seminar endocrine disrupters. 02.-04.04.2001 Berlin. Proceedings, 97-98

[51] Derksen JGM, van Eijnatten GM, Lahr J, van der Linde P, Kroon AGM (2002): Environmental effects of human pharmaceuticals The presence and risks, Publishers: Association of River Waterworks RIWA, Institute for Inland Water Management and Waste Water Treatment-RIZA, RIZA report 2001.051

[52] Länge R, Hutchinson ThH, Croudace ChP, Siegmund F, Schweinfurth H, Hampe P, Panter GH, Sumpter JP (2001): Effects of the synthetic estrogen $17 \alpha$-Ethinylestradiol on the life-cycle of the fathead minnow (Pimephales promelas). Environ Toxicol Chem (20) 6, 1216-1227

[53] Webb SF (2001): A data-based Perspective on the Environmental Risk Assessment of Human Pharmaceuticals I- Collation of Available Ecotoxicity Data, enthalten in: Kümmerer K (Hrsg.) (2001): Pharmaceuticals in the Environment - Fate, Effects and Risks. Springer Verlag Berlin, 265 pp

[54] Römbke J, Knacker T, Teichmann H (2001): Ecotoxicological Evaluation of Pharmaceuticals, entbalten in: Kümmerer K (Hrsg) (2001): Pharmaceuticals in the Environment - Fate, Effects and Risks. Springer Verlag Berlin, $265 \mathrm{pp}$

[55] EMEA (2003): The European Agency for the Evaluation of Medicinical Products, Note for Guidance on Environmental Risk Assessment of non - genetically modified Organism (non-GMO) containing Medicinical Products for Human Use. London. CPMP/SWP/ 4447/00 11 June 2003, 19 pp

[56] Ferrari B, Paxeus N, Lo Giudice R, Pollio A, Garrii J (2003): Ecotoxicological impact of Pharmaceuticals found in treated waste waters: study of carbamazepine, clofibric acid and diclofenac. Ecotox Environ Saf 55, 359-370

[57] Cleuvers M (2002): Aquatische Ökotoxikologie ausgewählter Arzneimittel. UWSF - Z Umweltchem Ökotox 14 (2) 85-89

[58] IMS/UBA (2002): IMS Health chemical country profile 2002/UBA Forschungsvorhaben FKZ 20067401

Eingereicht: 17. April 2003 Akzeptiert: 31. Dezember 2003 OnlineFirst: 09. Februar 2004 